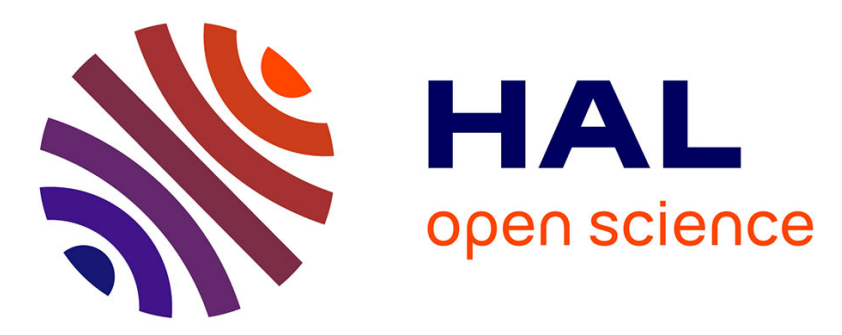

\title{
Characterization of partial derivatives with respect to material parameters in a fluid-solid interaction problem
}

Izar Azpiroz, Hélène Barucq, Rabia Djellouli, Ha Pham

\section{To cite this version:}

Izar Azpiroz, Hélène Barucq, Rabia Djellouli, Ha Pham. Characterization of partial derivatives with respect to material parameters in a fluid-solid interaction problem. Journal of Mathematical Analysis and Applications, 2018, 465, pp.903-927. 10.1016/j.jmaa.2018.05.046 . hal-01806939

\section{HAL Id: hal-01806939 \\ https://hal.science/hal-01806939}

Submitted on 6 Jun 2018

HAL is a multi-disciplinary open access archive for the deposit and dissemination of scientific research documents, whether they are published or not. The documents may come from teaching and research institutions in France or abroad, or from public or private research centers.
L'archive ouverte pluridisciplinaire HAL, est destinée au dépôt et à la diffusion de documents scientifiques de niveau recherche, publiés ou non, émanant des établissements d'enseignement et de recherche français ou étrangers, des laboratoires publics ou privés. 


\title{
Characterization of partial derivatives with respect to material parameters in a fluid-solid interaction problem.
}

\author{
Izar Azpiroz ${ }^{\mathrm{a}}$, Hélène Barucq ${ }^{\mathrm{a}}$, Rabia Djellouli ${ }^{\mathrm{b}}, \mathrm{Ha}$ Pham $^{\mathrm{a}, *}$ \\ ${ }^{a}$ Inria Bordeaux Sud-Ouest Research Center, Project-Team Magique-3D, LMA - UMR CNRS \\ 5142 - E2S, Université de Pau et des pays de l'Adour, France. \\ ${ }^{b}$ IRIS, Department of Mathematics, California State University, Northridge.
}

\begin{abstract}
For a fluid-solid interaction problem with Lipschitz interface, we investigate the partial Fréchet differentiability of the solutions and the approximate far-fieldpattern with respect to solid material parameters. Differentiability is shown in standard Sobolev framework, and the derivatives are characterized as solutions to inhomogeneous fluid-solid transmission problems. To validate the accuracy of the characterization, we compare analytical values with numerical ones given by Interior Penalty Discontinuous Galerkin (IPDG) in a setting with circular obstacles. Our comparisons also show that IPDG gives results with high precision and incurs almost no effect of discretization error accumulation.
\end{abstract}

Keywords: sensitivity with respect to Lamé parameters, partial Fréchet derivative, fluid-solid interaction, approximate far-field-pattern.

\section{Introduction}

In this work, for a fluid-solid interaction problem in $\mathbb{R}^{m}, m=2,3$, with Lipschitz fluid-solid interface, we investigate the partial Fréchet differentiability of the solutions and the approximate far-field-pattern (a-FFP) with respect to the solid material parameters. These include the Lamé parameters $\lambda, \mu$ and the solid density $\rho_{\mathrm{s}}$. The original fluid-solid interaction denoted by $\mathbf{O P} \mathbf{P}_{\infty}$ is posed in infinite space, $c f$. Figure 1(a), For numerical evaluation using volumed-based discretization, the infinite domain is truncated using absorbing boundary condition (ABC), and the problem we focus on, denoted by $\mathbf{O P}$, is defined on a finite convex domain $\Omega_{\text {finite }}$,

${ }^{*}$ Corresponding author

Email addresses: izar.azpiroz@inria.fr (Izar Azpiroz), helene.barucq@inria.fr (Hélène Barucq), rabia.djellouli@csun.edu (Rabia Djellouli), ha.howard@inria.fr (Ha Pham) 
$c f$. Figure 1(b). Because of domain truncation, we work with the approximated farfield-pattern (a-FFP), $c f$. (36), instead of the true far-field-pattern (FFP), $c f$. (11). Although defined on a finite domain, our problem is in the category of transmission

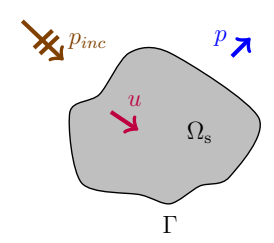

(a) Original prob$\operatorname{lem} \mathbf{O P}_{\infty}$ on $\mathbb{R}^{m}$.

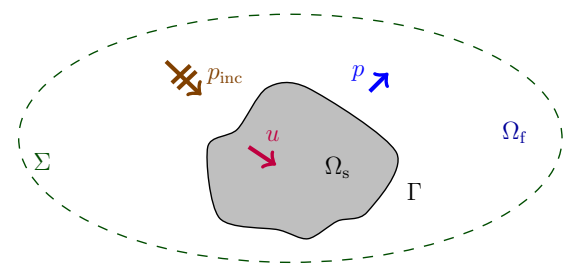

(b) (Truncated) problem OP on the bounded region $\Omega_{\text {finite }}=\Omega_{\mathrm{s}} \cup \Omega_{\mathrm{f}}$.

Figure 1: In fluid-solid interaction problems, an incident wave is diffracted by an immersed solid body, and generates scattered fluid pressure $p$ and transmitted solid displacement $u$.

scattering with penetrable obstacles 1 , to be distinguished with elastic problems on bounded domains such as [8] for elastostatics, [9] in time-harmonic elastodynamics, or [10, 11] in elastodynamics.

For problem $\mathrm{OP}$, we carry out the following tasks. We first extend the wellposedness results in [12] for a generic family of ABCs (Task 1). We then introduce the auxiliary problems and make sense of their PDE forms in the 'standard' Sobolev framework (see Notations in subsection 2.1), and study their wellposedness via variational formulation (Task 2). We next show the separate continuity and partial Fréchet differentiability of the solution operator and a-FFP (associated to $\mathbf{O P}$ ), and characterize their partial derivatives as solutions to the auxiliary problems (Task 3). This justifies their numerical evaluation by discretizing the corresponding variational formulations. The accuracy of this characterization is validated by an analytical-numerical comparison, using IPDG [13], for a setting with circular obstacle and circular artificial boundary (Task 4).

We limit ourselves to showing partial differentiability, which is sufficient for iterative inversion procedures which reconstruct separately each material parameter ${ }^{2}$. What concerns us more is the Lipschitz interface. Note that most references assume at least $\mathcal{C}^{2}$ regularity, $c f$. [6, 2], with the exception of [5] for acoustic scattering with Lipschitz obstacles, and [14, 15, 16] for acoustic-elastic transmission (in fact $\mathbf{O P}_{\infty}$ ) with polygonal-shaped obstacles, and the aforementioned [11, 8]

\footnotetext{
${ }^{1}$ In the category of penetrable obstacles, our problem studies acoustic-elastic transmission, to be distinguished with acoustic-acoustic $c f$. [1] and elastic-elastic $c f$. 22. This is contrast with impenetrable obstacles, e.g. [3, 4, 5] in acoustics, and [6, 7] in elastics.

${ }^{2}$ Joint continuity and Fréchet differentiability can be obtained with slight modification of the current work.
} 
for elasticity on bounded Lipschitz domain. Under Lipschitz assumption, the auxiliary problems can contain singular boundary or interface terms that do not fit in the canonical boundary Sobolev spaces, and to give sense to the problem is not trivial ( $c f$. [14] and further discussion in subsection 2.3). Furthermore, for fluidsolid problems, existence of solutions is not always guaranteed due to the existence of solid resonance modes called Jones frequencies (defined in (26)).

The main feature of our work is that we make sense of the auxiliary problem in its PDE form within the standard Sobolev framework, then prove its equivalence to the variational formulation ${ }^{3}$. These justifications seem to be overlooked in literature, when one works directly with the variational forms, e.g. the aforementioned references [10, 11, 8], cf. Remark 5. Although [14, 15, 16] also study the auxiliary problems in PDE form, they use implicit function theorem to show differentiability, while we work directly with the solution operator and use standard analysist Other approaches to show Fréchet differentiability can be e.g. boundary integral operator, and factorization technique. Note that the literature investigating this topic is mostly concerned with domain derivatives 5 .

The rest of the paper contains two parts, mathematical analysis is in Section 2, and the numerical experiments in Section 3. The main results of Task 1 are in Theorem 2 and Corollary 3 , those of Task 2 in Proposition 5 and Corollary 6, and those of Task 3 in Proposition 8 and Corollary 9. The analysis for solid density $\rho_{\mathrm{s}}$ is straightforward and follows from the same analysis, with only results listed in subsection 2.5.

\section{Mathematical analysis}

\subsection{Notations}

Geometry. In Figure 1(b), we have introduced the solid obstacle $\Omega_{s}$ with Lipschitz boundary $\Gamma$, the finite convex domain $\Omega_{\text {finite }}$ with exterior boundary $\Sigma$, and the finite fluid region $\Omega_{\mathrm{f}}$. We have $\Omega_{\mathrm{f}}=\left(\mathbb{R}^{m} \backslash \overline{\Omega_{\mathrm{s}}}\right) \cap \Omega_{\text {finite }}$. Along any closed curve, the sign convention for the unit normal vector ${ }^{6} \nu$ is outward.

\footnotetext{
${ }^{3}$ We work with the original PDE form which gives physical meaning to the solutions. Variational formulation is used for well-posedness and for numerical evaluation.

${ }^{4}$ i.e. by showing that the operators verify the definition of continuity and differentiability.

${ }^{5}$ We cite some representative references which study domain derivatives in obstacle scattering. Using boundary integral operator in acoustics cf. [17], elastics [2], electromagnetics [18, 19, 20]. Using factorization technique by [21, in elastics $c f$. [6], acoustics and electromagnetics [22]. Using implicit function theorem for acoustics $c f$. [23, [5], and acoustic-elastic transmission [14, 15, 16]. Using variational methods in acoustics $c f$. [3, 1, and elastics [7, 2]. The factorization technique is more adapted to inversion with Linear Sampling method, $c f$. [6, 24] for discussion.

${ }^{6}$ By definition, a Lipschitz boundary can be represented locally as a graph of a Lipschitz function. Such a boundary inherits a (surface) measure $\sigma$ and a $L^{\infty}$ unit normal vector $\nu$ which
} 
Media. The fluid is assumed to be homogeneous compressible inviscid with constant density $\rho_{\mathrm{f}}$ and sound velocity $\mathrm{c}_{\mathrm{f}}$. The solid is supposed to be linearly isotropic homogeneous elastic of constant density $\rho_{\mathrm{s}}$. This means its constitutive law relating the stress tensor $\boldsymbol{\sigma}$ to the strain tensor $\boldsymbol{\epsilon}(u)$,

$$
\boldsymbol{\epsilon}(u) \triangleq \frac{\nabla u+(\nabla u)^{t}}{2},
$$

is given by linear Hooke's law

$$
\boldsymbol{\sigma}(u)=\mathbf{C}: \boldsymbol{\epsilon}(u)
$$

with the elastic stiffness tensor $\mathbf{C}$ invariant under rotations and reflections,

$$
\mathbf{C}_{i j k l}=\lambda \delta_{i j} \delta_{k l}+\mu \delta_{i l} \delta_{j k} \quad, \quad 1 \leq i, j, k, l \leq m .
$$

Here $\lambda$ and $\mu$ are the Lamé parameters. The material is in addition assumed to be pure elastic, i.e. $\rho_{\mathrm{s}}, \lambda, \mu \in \mathbb{R}, c f$. [27]. We use the dot $\cdot$ and double dot : notation to denote single and double contraction, $c f$. [28]. For tensor $\mathbf{C}=\left(\mathbf{C}_{i j k l}\right)$ of order 4 , matrix $\mathbf{a}=\left(\mathbf{a}_{i j}\right)$, and vector $u=\left(u_{i}\right),(\mathbf{C}: \mathbf{a})_{i j}=\sum_{k, l} \mathbf{C}_{i j k l} \mathbf{a}_{i j} ;(\mathbf{a} \cdot u)_{i}=\sum_{j} \mathbf{a}_{i j} u_{j}$. Function spaces.

- For a function space $\mathbb{F}$, denote by $\mathbb{F}^{\prime}$ its dual, i.e. the set of bounded linear functionals from $\mathbb{F}$ to $\mathbb{C}$.

- Following from the usual convention, $\mathcal{D}(\Omega)$ is the space of smooth functions with compact support in domain $\Omega$.

- For $s \in \mathbb{R}, H^{s}\left(\mathbb{R}^{m}\right)$ is the usual Sobolev space on $\mathbb{R}^{m}$, cf. [25, p.76].

- We follow [25, p.77] to define the Sobolev space for a bounded domain $\Omega \subset \mathbb{R}^{m}$,

$$
H^{s}(\Omega) \triangleq\left\{u \in \mathcal{D}^{\prime}(\Omega): u=\left.U\right|_{\Omega} \text { for some } U \in H^{s}\left(\mathbb{R}^{m}\right)\right\}, s \in \mathbb{R}^{+}
$$

- For a Lipschitz boundary $\Lambda$, the Sobolev spaces $H^{s}(\Lambda)$ are well-defined for $|s| \leq 1$ cf. [25, p.98], having the property $H^{-s}(\Lambda)=\left(H^{s}(\Lambda)\right)^{\prime}$.

- We denote $\langle\cdot, \cdot\rangle_{s, \Lambda}$ as the duality pairing between $H^{-s}(\Lambda)$ and $H^{s}(\Lambda)$ with pivot $L^{2}(\Lambda)$, with convention

$$
\langle f, \phi\rangle_{s, \Lambda}=(f, \phi)_{L^{2}(\Lambda)}=\int_{\Lambda} f \bar{\phi} \mathrm{d} s
$$

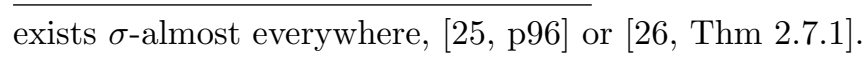


- Following [25, p.106], vector- or matrix-valued Sobolev spaces are written as,

$$
\begin{gathered}
H^{s}(\Omega)^{m}=H^{s}\left(\Omega ; \mathbb{C}^{m}\right) \quad, \quad H^{s}(\Omega)^{m \times m}=H^{s}\left(\Omega ; \mathbb{C}^{m \times m}\right) \quad, \quad s \in \mathbb{R}^{+} \\
H^{s}(\Lambda)^{m}=H^{s}\left(\Lambda, \mathbb{C}^{m}\right),|s| \leq 1
\end{gathered}
$$

- Our framework for well-posedness is the Gelfand triple $\mathbf{H} \subset \mathbf{U} \subset \mathbf{H}^{\prime}$,

$$
\mathbf{H} \triangleq H^{1}\left(\Omega_{\mathrm{f}}\right) \times H^{1}\left(\Omega_{\mathrm{s}}\right)^{m} \quad ; \quad \mathbf{U} \triangleq L^{2}\left(\Omega_{\mathrm{f}}\right) \times L^{2}\left(\Omega_{\mathrm{s}}\right)^{m}
$$

Trace operator. For $\frac{1}{2}<s \leq 1$, the trace operator $\gamma_{0}: \mathcal{D}(\bar{\Omega}) \rightarrow \mathcal{D}(\partial \bar{\Omega}), u \mapsto \gamma_{0} u=$ $\left.u\right|_{\partial \bar{\Omega}}$ has a unique extension to a bounded operator from $H^{s}(\Omega) \rightarrow H^{s-1 / 2}(\partial \bar{\Omega})$, with a continuous right inverse, see [25, Thm 3.37].

Conormal derivative. The conormal derivative operator $\frac{\partial}{\partial \nu}: \mathcal{D}(\bar{\Omega}) \rightarrow \mathcal{D}(\partial \bar{\Omega})$, $q \mapsto \frac{\partial q}{\partial \nu}=\gamma_{0}(\nabla q) \cdot \nu$ can be extended to a bounded map from $\left\{q \in H^{1}(\Omega) \mid \Delta q \in\right.$ $\left.\left(H^{1}(\Omega)\right)^{\prime}\right\} \longrightarrow H^{-1 / 2}(\partial \bar{\Omega}), c f$. [26, Thm 2.7 .7 p69]. In particular, if $p \in H^{1}\left(\Omega_{\mathrm{f}}\right)$ satisfies $\Delta p+\kappa^{2} p=0$ in $L^{2}\left(\Omega_{\mathrm{f}}\right)$, its conormal derivative is well-defined with $\frac{\partial}{\partial \nu} p \in$ $H^{-1 / 2}(\Gamma)$, and we have the integration-by-parts (IP) identity: for $q \in H^{1}\left(\Omega_{\mathrm{f}}\right)$,

$$
\int_{\Omega_{\mathrm{f}}}-(\Delta p) \bar{q} \mathrm{~d} x=\int_{\Omega_{\mathrm{f}}}(\nabla p \cdot \nabla \bar{q}) \mathrm{d} x+\left\langle\frac{\partial p}{\partial \nu}, q\right\rangle_{1 / 2, \Gamma}-\left\langle\frac{\partial p}{\partial \nu}, q\right\rangle_{1 / 2, \Sigma} .
$$

Normal trace. Following [29], the normal trace operator for matrices

$$
\gamma_{n}: \mathcal{D}(\bar{\Omega})^{m \times m} \rightarrow \mathcal{D}(\partial \bar{\Omega})^{m} \quad, \quad A \mapsto A \cdot \nu
$$

can be extended to a continuous map from

$$
\mathcal{H}^{0}(\operatorname{div}, \Omega) \triangleq\left\{\mathbf{v} \in L^{2}(\Omega)^{m \times m} \mid \operatorname{div} \mathbf{v} \in L^{2}(\Omega)^{m}\right\} \quad \longrightarrow \quad H^{-1 / 2}(\partial \bar{\Omega})
$$

by using density arguments and the Green's identity,

$$
\int_{\Omega}(\operatorname{div} \boldsymbol{u}) \cdot v \mathrm{~d} x=-\int_{\Omega} \boldsymbol{u}: \nabla \boldsymbol{v} \mathrm{d} x+\int_{\partial \bar{\Omega}}(\boldsymbol{u} \cdot \nu) \cdot v \mathrm{~d} s \quad, \quad \boldsymbol{u}, \boldsymbol{v} \in \mathcal{D}(\bar{\Omega})^{m \times m} .
$$

\subsection{Fluid-solid transmission problems}

In this part, we realize Task 1 and study the well-posedness for OP. We also provide a bound for the norm of the solution operator with an explicit bounding constant. This estimate will be used in a later subsection to show separate continuity and partial differentiability.

Original problem. When the configuration is excited by an exterior incident wave $p_{\text {inc }}(x)$ of frequency $\omega / 2 \pi, c f$. Figure $1(\mathrm{a})$, the scattered fluid pressure $p$ and the 
transmitted solid displacement $u$ satisfy the following transmission problem in $\mathbb{R}^{m}$, with the fluid wavenumber $\kappa=\frac{\omega}{\mathrm{c}_{\mathrm{f}}}$,

$$
\left(\mathbf{O P}_{\infty}\right)\left\{\begin{array}{cr}
\operatorname{div} \boldsymbol{\sigma}+\omega^{2} \rho_{\mathrm{s}} u=0 & \text { in } L^{2}\left(\Omega_{\mathrm{s}}\right)^{m} \\
\Delta p+\kappa^{2} p=0 & \text { in } L^{2}\left(\Omega_{\mathrm{f}}\right) \\
\boldsymbol{\sigma} \cdot \nu+p \nu=-p_{\text {inc }} \nu & \text { in } H^{-1 / 2}(\Gamma)^{m} \\
\omega^{2} \rho_{\mathrm{f}} u \cdot \nu-\frac{\partial p}{\partial \nu}=\frac{\partial p_{\text {inc }}}{\partial \nu} & \text { in } H^{-1 / 2}(\Gamma) \\
\lim _{r \rightarrow+\infty} r^{\frac{m-1}{2}}\left(\partial_{r} p-\mathrm{i} \kappa p\right)=0 & r=|x| .
\end{array}\right.
$$

Propagation in the fluid is governed by the Helmholtz equation (7), and in the solid by the time-harmonic elastodynamic equation (6). The interaction between the fluid and the solid is described by the kinematic interface condition (8), and the dynamic one (9), both defined along the interface $\Gamma$. To guarantee uniqueness of solutions, the outgoing Sommerfeld radiation condition (10) is imposed at infinity.

The scattered fluid pressure in $\mathbf{O P}_{\infty}$ has the asymptotic behavior of an outgoing spherical wave, $c f$. [30, Thm 2.6],

$$
p(x)=\frac{e^{\mathrm{i} \kappa|x|}}{|x|^{(m-1) / 2}}\left(p_{\infty}(\hat{x})+\mathrm{O}\left(|x|^{-1}\right)\right), \quad|x| \rightarrow \infty ; \hat{x}=\frac{x}{|x|} .
$$

In the above expression, $p_{\infty}$ is called the far-field pattern and satisfies the identity,

$$
p_{\infty}(\hat{x})=\mathfrak{c}(m) \int_{\tilde{\Gamma}} \frac{\partial e^{-\mathrm{i} \kappa \hat{x} \cdot y}}{\partial \nu(y)}\left(\gamma_{0} q\right) \mathrm{d} s(y)-\mathfrak{c}(m)\left\langle\frac{\partial q}{\partial \nu}, e^{\mathrm{i} \kappa \hat{x} \cdot y}\right\rangle_{1 / 2, \tilde{\Gamma}},
$$

for any simple (at least Lipschitz) closed curve $\tilde{\Gamma}$ enclosing $\Omega_{\mathrm{s}}$, i.e. $\tilde{\Gamma} \subset \overline{\mathbb{R}^{m} \backslash \Omega_{\mathrm{s}}}$, and with the defined constant

$$
\mathfrak{c}(m) \triangleq\left\{\begin{array}{ll}
\frac{e^{\mathrm{i} \pi / 4}}{8 \pi \kappa} & , m=2 \\
\frac{1}{4 \pi} & , m=3
\end{array} .\right.
$$

Truncated problem. For numerical computations, the region exterior to the solid is truncated to the finite domain $\Omega_{\text {finite }}, c f$. Figure $1(\mathrm{~b})$. The radiation condition (10) is replaced by absorbing boundary conditions (ABC) along $\Sigma$. We work with those of Robin type $\frac{\partial p}{\partial \nu}=-\mathscr{B} \gamma_{0} p$ in $H^{-1 / 2}(\Sigma)$, where operator $\mathscr{B}$ satisfies assumptions,

$$
\begin{gathered}
\mathscr{B}: H^{1 / 2}(\Sigma) \rightarrow H^{-1 / 2}(\Sigma) \quad \text { is bounded; } \\
\operatorname{Re}\langle\mathscr{B} \gamma, \gamma\rangle_{1 / 2, \Sigma} \geq 0, \operatorname{Im}\langle\mathscr{B} \gamma, \gamma\rangle_{1 / 2, \Sigma}<0, \text { for all } \gamma \neq 0 \text { in } H^{1 / 2}(\Sigma) .
\end{gathered}
$$


The resulting problem is denoted by $\mathbf{O P} \mathbf{P}_{\mathscr{B}}$ (or simply $\mathbf{O P}$ for generic operator $\mathscr{B}$ ),

$$
(\mathbf{O P})\left\{\begin{array}{rlrl}
\operatorname{div} \boldsymbol{\sigma}+\omega^{2} \rho_{\mathrm{s}} u & =0 & & \text { in } L^{2}\left(\Omega_{\mathrm{s}}\right)^{m} \\
\Delta p+\kappa^{2} p & =0 & \text { in } L^{2}\left(\Omega_{\mathrm{f}}\right) \\
\boldsymbol{\sigma} \cdot \nu+p \nu & =-p_{\text {inc }} \nu & & \text { in } H^{-1 / 2}(\Gamma)^{m} \\
\omega^{2} \rho_{\mathrm{f}} u \cdot \nu-\frac{\partial p}{\partial \nu} & =\frac{\partial p_{\text {inc }}}{\partial \nu} & & \text { in } H^{-1 / 2}(\Gamma) \\
\frac{\partial p}{\partial \nu} & =-\mathscr{B} p & & \text { in } H^{-1 / 2}(\Sigma) .
\end{array}\right.
$$

Assumptions (14) are needed for well-posedness, see discussion below. For the problem $\mathrm{OP}$ to be a good approximation of the original one $\mathbf{O P}_{\infty}$, operator $\mathscr{B}$ is chosen to be the exterior Dirichlet-to-Neumann map (D-t-N) T, see [31, Section 3.1 .2 p.63] for definition, or a good approximation of this.

- When $\Sigma$ is a circle, we can take $\mathscr{B}$ as $\mathbf{T}$, as done in [12]. With this choice, the $\mathrm{ABC}$ is exact, i.e. the original problem $\mathrm{OP}_{\infty}$ is equivalent to $\mathrm{OP}$, cf. [12, Thm 1]. The resulting problem is referred to as $\mathbf{O P}_{\mathbf{T}}$.

- Under the general assumption that $\Sigma$ is convex, we use the classical zero-th and first order absorbing boundary condition 7 , in which $\mathscr{B}$ equals to operators

$$
\mathbf{T}_{0} \triangleq-\mathrm{i} \kappa \quad, \quad \mathbf{T}_{1} \triangleq-\mathrm{i} \kappa+\frac{\varkappa_{\Sigma}}{2},
$$

respectively. The resulting problems are denoted by $\mathbf{O P}_{\mathbf{T}_{0}}$ and $\mathbf{O P}_{\mathbf{T}_{1}}$. Here, $\varkappa_{\Sigma}$ is the curvature of the curve $\Sigma$ when $m=2$, and $\frac{\varkappa_{\Sigma}}{2}$ is the mean curvature of the surface $\Sigma$ when $m=3$.

Lemma 1. The following choices of $\mathscr{B}$ satisfy properties (14): $\mathscr{B}=\mathbf{T}$ when $\Sigma$ is spherical (or circular), $\mathscr{B}=\mathbf{T}_{0}$ for general $\Sigma$, or $\mathscr{B}=\mathbf{T}_{1}$ when $\Sigma$ is convex with bounded positive curvature.

Proof. We have $\mathbf{T}: H^{1 / 2}(\Sigma) \rightarrow H^{-1 / 2}(\Gamma)$ is bounded with a bounded inverse ${ }^{8}$, and satisfies (14), cf. [12, Thm 1 and Lem 3] or [31, Section 3.2] for $m=3$, and [34, Thm 5.22] for $\mathrm{m}=2$.

\footnotetext{
${ }^{7}$ For the derivation of these conditions, see e.g. [32, Eqn 21] for $m=3$ and [33, Eqn 1.31] for $m=2$. These conditions are also listed in [31, Section 3.3.3] for circular $\Sigma$; in this case $\varkappa_{\Sigma}=R^{-1}$, with $R$ the radius of $\Sigma$.

8 The sign convention for $\mathbf{T}$ in 34 is different from [12, 31]. In either convention, the properties of $\mathbf{T}$ are shown by using the multipole expansions of solutions to the Helmholtz equation. For the boundedness of $\mathbf{T}$ on general curves or surfaces, see [30, Thm 3.13] for $\mathcal{C}^{2}$ boundaries, and [35, Thm 1.4] for nontrapping polygons and star-shaped Lipschitz domains.
} 
The boundedness for $\mathbf{T}_{0}$ follows immediately from the fact that it is a multiple of the identity. That for $\mathbf{T}_{1}$ follows additionally from the boundedness of the curvature of $\Sigma$. We next verify the second property in (14). For $\phi \in H^{1 / 2}(\Sigma)$, we have

$$
\langle\mathscr{B} \phi, \phi\rangle_{1 / 2, \Sigma}=\int(\mathscr{B} \phi) \bar{\phi} \mathrm{d} x=\left\{\begin{array}{ll}
-\mathrm{i}\|\phi\|_{L^{2}(\Sigma)}^{2} & , \quad \mathscr{B}=\mathbf{T}_{0} \\
-\mathrm{i}\|\phi\|_{L^{2}(\Sigma)}^{2}+\int_{\Sigma} \frac{\varkappa_{\Sigma}}{2}|\phi|^{2} \mathrm{~d} s & , \quad \mathscr{B}=\mathbf{T}_{1}
\end{array} .\right.
$$

Under the assumption $\varkappa_{\Sigma}>0$, we have $\operatorname{Re}\langle\mathscr{B} \phi, \phi\rangle_{1 / 2, \Gamma} \geq 0$ and $\operatorname{Im}\langle\mathscr{B} \phi, \phi\rangle_{1 / 2, \Gamma} \leq$ 0. In addition, $\operatorname{Im}\langle\mathscr{B} \phi, \phi\rangle_{1 / 2, \Gamma}=0$ implies $\phi=0$.

Variational formulation. We follow [12] to introduce the following sesquilinear forms. The volume sesquilinear forms corresponding to the (reduced) elastodynamic equation in $\Omega_{\mathrm{s}}$ and the Helmholtz equation in $\Omega_{\mathrm{f}}$ are

$$
\begin{gathered}
\mathbf{a}_{\mathrm{s}}(u, v) \triangleq \int_{\Omega_{\mathrm{s}}} \boldsymbol{\sigma}(u): \nabla \bar{v} \mathrm{~d} x-\int_{\Omega_{\mathrm{s}}} \omega^{2} \rho_{\mathrm{s}} u \bar{v} \mathrm{~d} x ; u, v \in H^{1}\left(\Omega_{\mathrm{s}}\right)^{m}, \\
\mathbf{a}_{\mathrm{f}}(p, q) \triangleq \int_{\Omega_{\mathrm{f}}} \nabla p \cdot \nabla \bar{q} \mathrm{~d} x-\int_{\Omega_{\mathrm{f}}} \kappa^{2} p \bar{q} \mathrm{~d} x ; p, q \in H^{1}\left(\Omega_{\mathrm{f}}\right) .
\end{gathered}
$$

The boundary sesquilinear forms corresponding to the transmission conditions on the solid-fluid interface $\Sigma$ are

$$
\begin{aligned}
\mathfrak{b}\left[\left(\begin{array}{c}
p \\
u
\end{array}\right),\left(\begin{array}{c}
q \\
v
\end{array}\right)\right] & \triangleq\left\langle u \cdot \nu, \gamma_{0} q\right\rangle_{1 / 2, \Gamma} ;\left(\begin{array}{l}
p \\
u
\end{array}\right),\left(\begin{array}{l}
q \\
v
\end{array}\right) \in \mathbf{H} ; \\
\mathfrak{b}^{\star}\left[\left(\begin{array}{c}
p \\
u
\end{array}\right),\left(\begin{array}{c}
q \\
v
\end{array}\right)\right] & \triangleq \overline{\mathfrak{b}\left[\left(\begin{array}{l}
p \\
u
\end{array}\right),\left(\begin{array}{l}
q \\
v
\end{array}\right)\right]} ;\left(\begin{array}{l}
p \\
u
\end{array}\right),\left(\begin{array}{c}
q \\
v
\end{array}\right) \in \mathbf{H} .
\end{aligned}
$$

Note that $\mathfrak{b}^{\star}\left[\left(\begin{array}{c}q \\ v\end{array}\right),\left(\begin{array}{c}p \\ u\end{array}\right)\right]=\overline{\langle v \cdot \nu, p\rangle_{1 / 2, \Gamma}}=\langle p, v \cdot \nu\rangle_{1 / 2, \Gamma}=\langle p \nu, v\rangle_{1 / 2, \Gamma}$. The sesquilinear form associated to the absorbing boundary condition along $\Sigma$ is,

$$
\boldsymbol{t}(p, q) \triangleq\left\langle\mathscr{B} \gamma_{0} p, \gamma_{0} q\right\rangle_{1 / 2, \Gamma}
$$

The main sesquilinear form in all of our variational problems is

$$
\begin{aligned}
\mathbf{a}\left[\left(\begin{array}{c}
p \\
u
\end{array}\right),\left(\begin{array}{c}
q \\
v
\end{array}\right)\right] & \triangleq \mathbf{a}_{\mathbf{s}}(u, v)+\mathfrak{b}^{\star}\left[\left(\begin{array}{c}
q \\
v
\end{array}\right),\left(\begin{array}{c}
p \\
u
\end{array}\right)\right] \\
& +\frac{1}{\omega^{2} \rho_{\mathrm{f}}} \mathbf{a}_{\mathrm{f}}(p, q)+\mathfrak{b}\left[\left(\begin{array}{c}
p \\
u
\end{array}\right),\left(\begin{array}{l}
q \\
v
\end{array}\right)\right]+\frac{1}{\omega^{2} \rho_{\mathrm{f}}} \boldsymbol{t}(p, q) ;\left(\begin{array}{c}
p \\
u
\end{array}\right),\left(\begin{array}{c}
q \\
v
\end{array}\right) \in \mathbf{H} .
\end{aligned}
$$

Denote by $\mathscr{A}=\mathscr{A}\left(\lambda, \mu, \rho_{\mathrm{s}}\right)$ the operator associated to a at $\left(\lambda, \mu, \rho_{\mathrm{s}}\right)$.

Well-posedness discussion. The well-posedness of $\mathbf{O P}_{\infty}$ and $\mathbf{O P}_{\mathbf{T}}$ in the framework $\mathbf{H} \subset \mathbf{U} \subset \mathbf{H}^{\prime}$ is addressed in [12]. In this case $(\mathscr{B}=\mathbf{T})$, the sesquilinear form 
$\mathbf{a}$ is shown to satisfy the Gårding's inequality on $\mathbf{H}$ (i.e. $\mathbf{H}$-coercive with respect to $\mathbf{U}), c f .[12$, Proposition 6],

$$
\operatorname{Re} \mathbf{a}\left[\left(\begin{array}{c}
q \\
v
\end{array}\right),\left(\begin{array}{c}
q \\
v
\end{array}\right)\right]+c\left\|\left(\begin{array}{c}
q \\
v
\end{array}\right)\right\|_{\mathbf{U}}^{2} \quad \geq \quad \alpha\left\|\left(\begin{array}{c}
q \\
v
\end{array}\right)\right\|_{\mathbf{H}}^{2} \quad, \quad\left(\begin{array}{c}
q \\
v
\end{array}\right) \in \mathbf{H} .
$$

This allows applying the Fredholm principle, $c f$. [26, Thm 2.1.60] to study the wellposedness of the variational problem equivalent to $\mathbf{O P}_{\mathbf{T}}$. Even when existence (of solutions) is given, uniqueness is not guaranteed due to Jones modes defined as

$$
\begin{aligned}
& \omega \text { is called a Jones frequency and } \\
& u \neq 0, u \in H^{1}\left(\Omega_{\mathrm{s}}\right) \text { a Jones mode }
\end{aligned} \text { if }(u, \omega) \text { solves }\left\{\begin{array}{l}
\operatorname{div} \boldsymbol{\sigma}+\omega \rho_{\mathrm{s}} u=0 \text { in } \Omega_{\mathrm{s}} \\
\boldsymbol{\sigma} \cdot \nu=0 ; u \cdot \nu=0 \text { on } \Gamma
\end{array} .\right.
$$

We refer to [36] and the references therein for a detailed discussion of Jones modes.

We now extend the result of [12, Thm 7] to arbitrary operator $\mathscr{B}$ satisfying (14), and obtain the well-posedness for $\mathbf{O P}_{\mathscr{B}}$. The proof follows [12] with minor modification. The completely new feature is an energy estimate with an explicit bounding constant9. For the rest of the subsection, notations are simplified and reflect only the dependence with respect to $\lambda$ and $\mu$. In particular, we write $\mathscr{A}=\mathscr{A}(\lambda, \mu)$ instead of $\mathscr{A}\left(\lambda, \mu, \rho_{\mathrm{s}}\right)$.

Theorem 2. Consider the sesquilinear form a defined in (25) with operator $\mathscr{B}$ satisfying (14), and the material parameters satisfying

$$
\lambda>0, \quad \mu \geq 0, \omega>0, \quad \rho_{\mathrm{f}}>0, \quad \rho_{\mathrm{s}}>0 .
$$

For arbitrary functional $\mathbf{l} \in \mathbf{H}^{\prime}$, the generic variational problem

$$
\text { Find }\left(\begin{array}{l}
p \\
u
\end{array}\right) \in \mathbf{H} \text { so that } \mathbf{a}\left[\left(\begin{array}{c}
p \\
u
\end{array}\right),\left(\begin{array}{c}
q \\
v
\end{array}\right)\right]=\mathbf{l}\left[\left(\begin{array}{c}
q \\
v
\end{array}\right)\right], \forall\left(\begin{array}{l}
q \\
v
\end{array}\right) \in \mathbf{H}
$$

has the following dichotomy.

1. If $\omega$ is not a Jones frequency, there exists a unique solution for (28). In addition, the solution depends continuously on the right hand side, described by the energy bound: for a fixed $\rho_{\mathrm{s}}$, and in a small enough neighborhood of a pair $(\lambda, \mu)$ with $h$ satisfying 32 ,

$$
\begin{aligned}
\left\|\left(p_{\lambda+h, \mu}, u_{\lambda+h, \mu}\right)\right\|_{\mathbf{H}} & \leq 2\left\|\mathscr{A}(\lambda, \mu)^{-1}\right\|_{\mathcal{L}\left(\mathbf{H}^{\prime}, \mathbf{H}\right)}\|\mathbf{l}\|_{\mathbf{H}^{\prime}}, \\
\left\|\left(p_{\lambda, \mu+h}, u_{\lambda, \mu+h}\right)\right\|_{\mathbf{H}} & \leq 2\left\|\mathscr{A}(\lambda, \mu)^{-1}\right\|_{\mathcal{L}\left(\mathbf{H}^{\prime}, \mathbf{H}\right)}\|\mathbf{l}\|_{\mathbf{H}^{\prime}}
\end{aligned} .
$$

\footnotetext{
${ }^{9}$ Estimate (60) in [12, Thm 7] needs some clarification. It should hold with a different bounding constant (not 1 as stated) and under the assumption that $\omega$ is not a Jones frequency.
} 
2. If $\omega$ is a Jones frequency, then there exists a solution to (28) if and only if functional 1 satisfies the compatibility condition

$$
\mathbf{l}\left[\left(\begin{array}{l}
0 \\
v
\end{array}\right)\right]=0 \quad, \quad \forall \text { Jones mode } v .
$$

If (30) is satisfied, a solution $(p, u) \in \mathbf{H}$ to 28 exists, with the fluid part $p$ being unique, and the solid displacement $u$ being unique modulo Jones modes.

Remark 1. The assumption on the Lamé parameters is needed for the $H^{1}\left(\Omega_{\mathrm{s}}\right)^{m}$ coercitivity of $\mathbf{a}_{\mathrm{s}}$, see [12, Eqn (49)-(50)], see also [25, Thm 10.3].

Proof. Well-posedness statement: The proof is identical to that for [12, Thm 7] with the following modifications to adapt to a general $\mathscr{B}$.

- In the proof to show that a satisfies the Gårding inequality, Eqn (57) in [12] is replaced by the property $\operatorname{Re}\langle\mathscr{B} \gamma, \gamma\rangle_{1 / 2, \Sigma} \geq 0$.

- In the investigation of the uniqueness of the pressure fluid, we consider $(p, u) \in \mathbf{H}$ such that $\mathbf{a}\left(\left(\begin{array}{l}p \\ u\end{array}\right),\left(\begin{array}{l}p \\ u\end{array}\right)\right)=0$. This means that $(p, u) \in \mathbf{H}$ satisfies (15)-119) with $p_{\text {inc }}=0$. Eqn (63) in [12] is replaced with

$$
\operatorname{Im} \mathbf{a}\left(\left(\begin{array}{l}
p \\
u
\end{array}\right),\left(\begin{array}{l}
p \\
u
\end{array}\right)\right)=\operatorname{Im}\left\langle\mathscr{B} \gamma_{0} p, \gamma_{0} p\right\rangle_{1 / 2, \Sigma}=0 .
$$

From here, we use the third property in (14),

$$
\operatorname{Im}\langle\mathscr{B} \gamma, \gamma\rangle_{1 / 2, \Sigma}<0 \text {, for all } \gamma \in H^{1 / 2}(\Sigma), \gamma \neq 0,
$$

to imply that $\gamma_{0} p=0$. Combining with the boundary condition (18), we obtain $\frac{\partial p}{\partial \nu}=-\mathscr{B} \gamma_{0} q=0$. The rest of the proof uses analytic continuation principle and follows as in [12, Section 4.2.1] to show that $p=0$.

Energy estimate when $\omega$ is not a Jones frequency: At $(\lambda, \mu),(\lambda+h, \mu)$ and

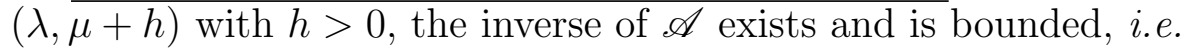

$$
\mathscr{A}(\lambda, \mu)^{-1}, \mathscr{A}(\lambda+h, \mu)^{-1}, \mathscr{A}(\lambda, \mu+h)^{-1} \in \mathcal{L}\left(\mathbf{H}^{\prime}, \mathbf{H}\right) .
$$

We will bound the norm of the last two inverses by that of $\mathscr{A}(\lambda, \mu)^{-1}$. Since the proof for $\mu+h$ is exactly the same, only that for $\lambda+h$ is written.

The map $(\lambda, \mu) \mapsto \mathscr{A}$ is affine separately in $\lambda$ and $\mu$, the only term that depends on $\lambda$ or $\mu$ is $\mathbf{a}_{\mathrm{s}}$. As a result, its partial derivative with respect to $\lambda$ is independent of $\lambda$, and we denote this by $\mathscr{D}_{1}(\mu) \in \mathcal{L}\left(\mathbf{H}, \mathbf{H}^{\prime}\right)$. We can write

$$
\begin{aligned}
\mathscr{A}(\lambda+h, \mu) & =\mathscr{A}(\lambda, \mu)+h \mathscr{D}_{1}(\mu) \\
& =\mathscr{A}(\lambda, \mu)_{\mathbf{H} \rightarrow \mathbf{H}^{\prime}}\left(\operatorname{Id}_{\mathbf{H} \rightarrow \mathbf{H}}-h \mathscr{A}(\lambda, \mu)_{\mathbf{H}^{\prime} \rightarrow \mathbf{H}}^{-1} \mathscr{D}_{1}(\mu)_{\mathbf{H} \rightarrow \mathbf{H}^{\prime}}\right) \\
\Rightarrow \mathscr{A}(\lambda+h, \mu)^{-1} & =(\mathrm{Id}-h \mathscr{K}(\lambda, \mu))^{-1} \mathscr{A}(\lambda, \mu)^{-1} .
\end{aligned}
$$


Here, we have defined the bounded operator $\mathscr{K}(\lambda, \mu)=\mathscr{A}(\lambda, \mu)^{-1} \mathscr{D}_{1}(\mu)$. When $0<h<\frac{1}{\mathfrak{c}(\lambda, \mu)}$, the inverse $(\operatorname{Id}+h \mathscr{K}(\lambda, \mu))^{-1}$ can be written as an operatorvalued Neumann series, and from this, we obtain the estimate

$$
\begin{gathered}
\left\|(\mathrm{Id}+h \mathscr{K}(\lambda, \mu))^{-1}\right\|_{\mathcal{L}(\mathbf{H})} \leq \frac{1}{1-h \mathfrak{c}(\lambda, \mu)}, \\
\text { with } \mathfrak{c}(\lambda, \mu) \triangleq\left\|\mathscr{A}(\lambda, \mu)^{-1}\right\|_{\mathcal{L}\left(\mathbf{H}^{\prime}, \mathbf{H}\right)}\left\|\mathscr{D}_{1}(\mu)\right\|_{\mathcal{L}\left(\mathbf{H}^{\prime}, \mathbf{H}\right)} .
\end{gathered}
$$

This is next used to bound,

$$
\left\|\mathscr{A}(\lambda+h, \mu)^{-1}\right\|_{\mathcal{L}\left(\mathbf{H}^{\prime}, \mathbf{H}\right)} \leq \frac{\left\|\mathscr{A}(\lambda, \mu)^{-1}\right\|}{1-h \mathfrak{c}(\lambda, \mu)} .
$$

One has the same estimate for $\mathscr{A}(\lambda, \mu+h)^{-1}$, with $\mathfrak{c}$ in 31 replaced by

$$
\mathfrak{d}(\lambda, \mu) \triangleq\left\|\mathscr{A}(\lambda, \mu)^{-1}\right\|_{\mathcal{L}\left(\mathbf{H}^{\prime}, \mathbf{H}\right)}\left\|\mathscr{D}_{2}(\lambda)\right\|_{\mathcal{L}\left(\mathbf{H}^{\prime}, \mathbf{H}\right)} .
$$

To obtain the announced estimates $(29)$, we impose further that

$$
h \mathfrak{c}(\lambda, \mu)<\frac{1}{2}, h \mathfrak{d}(\lambda, \mu)<\frac{1}{2} \Rightarrow \frac{1}{1-h \mathfrak{c}(\lambda, \mu)}<2, \frac{1}{1-h \mathfrak{d}(\lambda, \mu)}<2 .
$$

Remark 2. The compatibility condition (30) will be satisfied automatically, if $\mathbf{1}$ contains only boundary operators, which is the case for $\mathbf{O P}$.

Following the same reasoning as in [12, Thm 2], the transmission problem OP (15) - 19) is equivalent to the variational problem VOP,

$$
\text { Find }\left(\begin{array}{l}
p \\
u
\end{array}\right) \in \mathbf{H}: \mathbf{a}\left[\left(\begin{array}{c}
p \\
u
\end{array}\right),\left(\begin{array}{c}
q \\
v
\end{array}\right)\right]=\boldsymbol{l}_{\mathbf{O P}}\left[\left(\frac{\bar{q}}{v}\right)\right], \forall\left(\begin{array}{c}
q \\
v
\end{array}\right) \in \mathbf{H}
$$

with $\boldsymbol{l}_{\mathbf{O P}} \in \mathbf{H}^{\prime}, \boldsymbol{l}_{\mathbf{O P}}\left[\left(\begin{array}{c}q \\ v\end{array}\right)\right] \triangleq \frac{1}{\omega^{2} \rho_{\mathrm{f}}}\left\langle\frac{\partial p_{\text {inc }}}{\partial \nu}, \overline{\gamma_{0} q}\right\rangle_{1 / 2, \Gamma}-\left\langle p_{\text {inc }}, \overline{\gamma_{0} v}\right\rangle_{1 / 2, \Gamma},\left(\begin{array}{c}q \\ v\end{array}\right) \in \mathbf{H}$.

By Remark 2, functional $\boldsymbol{l}_{\text {OP }}$ satisfies the compatibility condition (30), see also [12, Thm 7]. As a result, existence of solutions for problem VOP is guaranteed in all cases. We summarize this result in the corollary below.

Corollary 3. With $\mathscr{B}$ satisfying (14) and the material parameters satisfying (27), there exists a solution $(p, u) \in \mathbf{H}$ to problem VOP, with $p$ unique. Additionally,

- if $\omega$ is a Jones frequency, the solid displacement $u$ is unique modulo Jones modes;

- if not, such $(p, u)$ is unique in $\mathbf{H}$, and in a neighborhood of $(\lambda, \mu)$ with $h$ satisfying (32), we have

$$
\begin{aligned}
\left\|\left(p_{\lambda+h, \mu}, u_{\lambda+h, \mu}\right)\right\|_{\mathbf{H}} & \leq \mathrm{c}(\lambda, \mu)\left\|p_{\mathrm{inc}}\right\|_{H^{2}\left(\Omega_{\mathrm{f}}\right)}, \\
\left\|\left(p_{\lambda, \mu+h}, u_{\lambda, \mu+h}\right)\right\|_{\mathbf{H}} & \leq \mathrm{c}(\lambda, \mu)\left\|p_{\text {inc }}\right\|_{H^{2}\left(\Omega_{\mathrm{f}}\right)},
\end{aligned}
$$

with a constant $\mathrm{c}(\lambda, \mu)>0$ independent of $h$. 
In the above estimates, we have used,

$$
\left\|\boldsymbol{l}_{\mathbf{O P}}\right\|_{\mathbf{H}^{\prime}}=\hat{C}\left(\left\|\frac{\partial p_{\text {inc }}}{\partial \nu}\right\|_{H^{-1 / 2}(\Gamma)}+\left\|p_{\text {inc }}\right\|_{H^{-1 / 2}(\Gamma)}\right) \leq \tilde{C}\left\|p_{\text {inc }}\right\|_{H^{2}\left(\Omega_{\mathrm{f}}\right)} .
$$

As a result of Corollary 3 , when $\omega$ is not a Jones frequency, we can define the solution operator

$$
\begin{gathered}
\mathcal{S}: \mathbb{R}_{+} \times \mathbb{R}_{+} \times \mathbb{R}_{+} \rightarrow \mathbf{H},\left(\lambda, \mu, \rho_{\mathrm{s}}\right) \mapsto \mathcal{S}\left(\lambda, \mu, \rho_{\mathrm{s}}\right)=\left(p_{\lambda, \mu, \rho_{\mathrm{s}}}, u_{\lambda, \mu, \rho_{\mathrm{s}}}\right), \\
\quad \text { with }\left(p_{\lambda, \mu, \rho_{\mathrm{s}}}, u_{\lambda, \mu, \rho_{\mathrm{s}}}\right) \text { the unique solution in } \mathbf{H} \text { to } 15-19 .
\end{gathered}
$$

Approximate far-field-pattern. Although $\mathbf{O P}$ is defined on a finite domain, by using (12), we can generalize the notion of far-field-pattern to this problem. From (12), we define the integral operator $\mathbf{I}$ : for $q \in H^{1}\left(\Omega_{\mathrm{f}}\right)$ with $\frac{\partial q}{\partial \nu} \in H^{-1 / 2}(\Gamma)$,

$$
(\mathbf{I} q)(\hat{x}) \triangleq \mathfrak{c}(m) \int_{\Gamma} \frac{\partial e^{-\mathrm{i} \kappa \hat{x} \cdot y}}{\partial \nu(y)}\left(\gamma_{0} q\right) \mathrm{d} s(y)-\mathfrak{c}(m)\left\langle\frac{\partial q}{\partial \nu}, e^{\mathrm{i} \kappa \hat{x} \cdot y}\right\rangle_{1 / 2, \Gamma}, \hat{x}=\frac{x}{|x|}
$$

Recall that $\mathfrak{c}(m)$ is defined in (13). The above definition exists in all cases, due to the uniqueness of the pressure field. Next, using the transmission conditions (17) and (18), we define the approximate FFP operator associated to OP as follows,

$$
\begin{aligned}
& \mathrm{F}: \mathbb{R}^{+} \times \mathbb{R}^{+} \times \mathbb{R}^{+} \longrightarrow \mathcal{C}^{\infty}\left(\mathbb{S}^{1}, \mathbb{C}\right),\left(\lambda, \mu, \rho_{\mathrm{s}}\right) \longrightarrow \mathrm{F}\left(\lambda, \mu, \rho_{\mathrm{s}}\right) \\
&\left(\mathrm{F}\left(\lambda, \mu, \rho_{\mathrm{s}}\right)\right)(\hat{x})= \mathfrak{c}(m) \int_{\Gamma}\left(\frac{\partial e^{-\mathrm{i} \kappa \hat{x} \cdot y}}{\partial \nu(y)} p-\left(\omega \rho_{\mathrm{f}}^{2} u \cdot \nu-\frac{\partial p_{\text {inc }}}{\partial \nu}\right) e^{-\mathrm{i} \kappa \hat{x} \cdot y}\right) \mathrm{d} s(y), \\
& \text { with }\left(p_{\lambda, \mu, \rho_{\mathrm{s}}}, u_{\lambda, \mu, \rho_{\mathrm{s}}}\right)=\mathcal{S}\left(\lambda, \mu, \rho_{\mathrm{s}}\right) .
\end{aligned}
$$

Remark 3. This approach to compute approximate far-field-patterns from near fields is also employed in [37]. It is more common to define $\mathrm{F}$ on a smooth curve $\tilde{\Gamma}$ outside of $\Omega_{\mathrm{s}}$ and not on the boundary of the obstacle. However, using the latter provides some computation advantages, e.g. the possibility to use (37), which involves only the zero-th order traces, and avoids having to calculate the conormal derivative of $p$ on a new curve.

\subsection{Auxiliary problem for partial derivatives with Lamé parameters}

We now introduce the auxiliary problems for Lamé parameters $\lambda$ and $\mu$. In general, the auxiliary problems take the same form as the original problem, however they are inhomogeneous. They contain volume and/or interface (boundary) sources for penetrable (impenetrable) obstacles, all defined in terms of the solution to the original problem and its (possibly higher order) traces ${ }^{10}$. Difficulty arises

\footnotetext{
10 For examples of the auxiliary problems studying domain derivatives of FPP in obstacle scattering, see [6, Thm 3.5 and 4.4 ] for elastic scattering, see [2, Thm 3.1] for elastic-elastic transmission, and see [3, Thm 2.1] for acoustic scattering.
} 
under low regularity of interfaces/boundaries, since interface (boundary) sources might not have well-defined traces in the canonical boundary spaces $H^{s}(\Gamma),|s| \leq 1$.

In our case, the auxilliary problem for the solid density contains only a volume source, while that for the Lamé parameters has additionally a singular interface source in the form of the normal trace of $\operatorname{div} A$ with $A \in L^{2}\left(\Omega_{\mathrm{s}}\right)^{m \times m}$, see also Remark 4. As such, we risk running into the same difficulty as [14] which studies domain derivatives for $\mathbf{O P}_{\infty}$. What differs in our problem is that the interface source is also the normal trace of the volume one, leading to cancellation in the more regular variational form, in which the 'troublesome' normal trace of $\operatorname{div} A$ no longer appears. This cancellation of singularities does not occur in the more difficult problem considered in [14] which only has interface sources ${ }^{11}$.

Remark 4. In our case, the above mentioned quantity A takes the form $\mathbf{C}^{\prime}: \boldsymbol{\epsilon}(u)$ where $u$ is the solid displacement from $\mathrm{OP}$. When $\Gamma$ is more regular, so is $A$, at least in $H^{1}\left(\Omega_{\mathrm{s}}\right)$, and its normal trace exists in $H^{1 / 2}(\Gamma)$ (using the mapping property of the trace operator $\gamma_{0}$ [25, Thm 3.38]). When $\Gamma$ is only Lipschitz, $u$ is in at most $H^{3 / 2}\left(\Omega_{\mathrm{s}}\right)^{m}$, cf. [12, Remark 2], and $A$ is in at most $H^{1 / 2}\left(\Omega_{\mathrm{s}}\right)^{m \times m}$.

As mentioned in the Introduction, instead of passing directly to the (more regular) variational formulation of the auxiliary problem, we first make sense of its PDE form, see also Remark 5. The main idea is to group together terms which do not apriori have well-defined normal traces when considered separately, but do when grouped together. We will need the following lemma which generalizes the normal trace of a matrix-valued distribution.

Lemma 4. If a matrix $A \in L^{2}\left(\Omega_{s}\right)^{m \times m}$ and $u \in H^{1}\left(\Omega_{\mathrm{S}}\right)^{m}$ satisfy

$$
\operatorname{div} \boldsymbol{\sigma}(u)+\omega^{2} \rho_{\mathrm{s}} u=-\operatorname{div} A \text { in }\left(H^{1}\left(\Omega_{s}\right)^{m}\right)^{\prime},
$$

then $(\boldsymbol{\sigma}+A) \in L^{2}\left(\Omega_{\mathrm{s}}\right)^{m \times m}$, and its normal trace is well-defined with $(\boldsymbol{\sigma}+A) \cdot \nu \in$ $H^{-1 / 2}(\Gamma)^{m}$. In addition, one has the generalized IP identity: for $v \in H^{1}\left(\Omega_{\mathrm{s}}\right)^{m}$,

$$
-\int_{\Omega_{\mathrm{s}}}[\operatorname{div}(\boldsymbol{\sigma}+A)] \cdot \bar{v} \mathrm{~d} x=\int_{\Omega_{\mathrm{s}}}(\boldsymbol{\sigma}+A) \cdot \nabla \bar{v} \mathrm{~d} x-\langle(\boldsymbol{\sigma}+A) \cdot \nu, v\rangle_{1 / 2, \Gamma} .
$$

Proof. We rearrange $(38)$ and obtain $\operatorname{div}(\boldsymbol{\sigma}+A)=-\omega^{2} \rho_{\mathrm{s}} u$ in $\left(H^{1}\left(\Omega_{\mathrm{s}}\right)^{m}\right)^{\prime}$. Since $u \in H^{1}\left(\Omega_{\mathrm{s}}\right)^{m}, \operatorname{div}(\boldsymbol{\sigma}+A) \in H^{1}\left(\Omega_{\mathrm{s}}\right)^{m}$ thus in $L^{2}\left(\Omega_{\mathrm{s}}\right)^{m}$. On the other hand, $u \in H^{1}\left(\Omega_{\mathrm{s}}\right)^{m}$, hence $\boldsymbol{\sigma}(u) \in L^{2}\left(\Omega_{\mathrm{s}}\right)^{m \times m}$ and thus $\boldsymbol{\sigma}(u)+A \in L^{2}\left(\Omega_{\mathrm{s}}\right)^{m \times m}$. This means $\boldsymbol{\sigma}+A \in \mathcal{H}^{0}\left(\operatorname{div}, \Omega_{\mathrm{s}}\right)$ defined in (4). By the discussion in subsection 2.1, its normal trace is well-defined in $H^{-1 / 2}(\bar{\Gamma})^{m}$, and the integration-by-parts formula can be generalized from the Green's identity (5).

\footnotetext{
${ }^{11}$ In [14, the regularity is increased to polygonal-shaped obstacles, and the Sobolev framework had to be enlarged, in particular, the exponent $-\frac{3}{2}$ was considered. The well-posedness of the resulting auxilliary problem remains an open question.
} 
From the above discussion, for $A \in L^{2}\left(\Omega_{\mathrm{s}}\right)^{m \times m}$ and $(p, u) \in \mathbf{H}$, we can define

$$
(\mathbf{D P})\left\{\begin{aligned}
\operatorname{div} \boldsymbol{\sigma}+\omega^{2} \rho_{\mathrm{s}} u & =-\operatorname{div} A & & \text { in }\left(H^{1}\left(\Omega_{\mathrm{s}}\right)^{m}\right)^{\prime} \\
\Delta p+\kappa^{2} p & =0 & & \text { in } L^{2}\left(\Omega_{\mathrm{f}}\right) \\
(\boldsymbol{\sigma}+A) \cdot \nu & =-p \nu & & \text { in } H^{-1 / 2}(\Gamma)^{m} \\
\omega^{2} \rho_{\mathrm{f}} u \cdot \nu-\frac{\partial p}{\partial \nu} & =0 & & \text { in } H^{-1 / 2}(\Gamma) \\
\frac{\partial p}{\partial \nu} & =-\mathscr{B} p & & \text { in } H^{-1 / 2}(\Sigma)
\end{aligned}\right.
$$

Remark 5. The quantity $\operatorname{div} A$ and its normal trace also appear in [8, Eqn (2.3)], and the right-hand-side of our variational formulation is the same as [8, Eqn (2.6)] and [11, Eqn (16),(17)]. Before passing to the variational formulation, [8] states the auxiliary problem in PDE form with the same assumed regularity of $A$, however without justifying its 'troublesome' normal trace. The PDE form of the auxiliary problem is not stated in [11]. Both works start their investigation directly with the variational formulation.

We now study the well-posedness for DP by first showing that it is equivalent to variational problem called VDP,

$$
\begin{gathered}
\text { Find }\left(\begin{array}{c}
p \\
u
\end{array}\right) \in \mathbf{H}: \mathbf{a}\left[\left(\begin{array}{c}
p \\
u
\end{array}\right),\left(\begin{array}{c}
q \\
v
\end{array}\right)\right]=\mathbf{l}_{\mathrm{fr} 1}\left[\left(\begin{array}{c}
\bar{q} \\
v
\end{array}\right)\right], \forall\left(\begin{array}{c}
q \\
v
\end{array}\right) \in \mathbf{H} ; \\
\text { with } \mathbf{l}_{\mathrm{fr} 1} \in \mathbf{H}^{\prime}, \mathbf{l}_{\mathrm{fr} 1}\left[\left(\begin{array}{c}
q \\
v
\end{array}\right)\right] \triangleq-\int_{\Omega_{\mathrm{s}}} A: \nabla v \mathrm{~d} x .
\end{gathered}
$$

Note that,

$$
\left\|\mathbf{l}_{\mathrm{fr} 1}\right\|_{\mathbf{H}^{\prime}}=\|A\|_{L^{2}\left(\Omega_{\mathrm{s}}\right)^{m \times m}} .
$$

Proposition 5 (Variational equivalence for DP). For matrix $A \in L^{2}\left(\Omega_{\mathrm{s}}\right)^{m \times m}$ and $(p, u) \in \mathbf{H}$, the following statements are equivalent.

(i) $(p, u)$ satisfies 40 -44.

(ii) $(p, u)$ is a solution of 45 .

Proof. (i) $\Rightarrow$ (ii): Apply a test function $v \in H^{1}\left(\Omega_{\mathrm{s}}\right)^{m}$ to both sides of (40), we obtain $-\left\langle\operatorname{div} \boldsymbol{\sigma}+\operatorname{div} A+\omega^{2} \rho_{\mathrm{s}} u, v\right\rangle_{1, \Omega_{\mathrm{s}}}=0$. By Lemma $4 \operatorname{div}(\boldsymbol{\sigma}+A) \in L^{2}\left(\Omega_{\mathrm{s}}\right)^{m}$, and the above equality can be written as

$$
-\int_{\Omega_{\mathrm{s}}}[\operatorname{div}(\boldsymbol{\sigma}+A)] \cdot \bar{v} \mathrm{~d} x-\omega^{2} \rho_{\mathrm{s}} \int_{\Omega_{\mathrm{s}}} u \cdot \bar{v} \mathrm{~d} x=0 .
$$

We next use the IP identity (39) from the same lemma, and transmission condition (42) to replace $(\boldsymbol{\sigma}+A) \cdot \nu$ with $p \nu$, and obtain

$$
\int_{\Omega_{\mathrm{s}}} \boldsymbol{\sigma}: \nabla \bar{v} \mathrm{~d} x+\langle p \nu, v\rangle_{1 / 2, \Gamma}-\omega^{2} \rho_{\mathrm{s}} \int_{\Omega_{\mathrm{s}}} u \cdot \bar{v} \mathrm{~d} x=-\int_{\Omega_{\mathrm{s}}} A: \nabla \bar{v} \mathrm{~d} x, \forall v \in H^{1}\left(\Omega_{\mathrm{s}}\right)^{m} .
$$


Here $\boldsymbol{\sigma}$ and $A$ can be written separately, since each is in $L^{2}\left(\Omega_{\mathrm{s}}\right)^{m \times m}$. In the terms of the sesquilinear forms defined in (21) and (23), the above equality is written as

$$
\mathbf{a}_{\mathrm{s}}(u, v)+\mathfrak{b}^{\star}\left[\left(\begin{array}{c}
q \\
v
\end{array}\right),\left(\begin{array}{l}
p \\
u
\end{array}\right)\right]=\mathrm{l}_{\mathrm{fr} 1}(\bar{v}), \forall(q, v) \in \mathbf{H} .
$$

In dealing with the fluid part, the proof is more standard. By using IP identity (3) and transmission condition (43) and ABC (44), we obtain

$$
\mathbf{a}_{\mathbf{f}}(p, q)+\omega^{2} \rho_{\mathrm{f}} \mathfrak{b}\left[\left(\begin{array}{c}
q \\
v
\end{array}\right),\left(\begin{array}{l}
p \\
u
\end{array}\right)\right]+\boldsymbol{t}(p, q)=0, \forall(q, v) \in \mathbf{H} .
$$

For more details, see also [12, Eqn (15)-(20)]. Statement (ii) is obtained by adding (47) to 48).

(ii) $\Rightarrow$ (i): Suppose $(p, u) \in \mathbf{H}$ solves 45

$$
\mathbf{a}\left[\left(\begin{array}{l}
p \\
u
\end{array}\right),\left(\begin{array}{l}
q \\
v
\end{array}\right)\right]=\mathbf{l}_{\mathrm{fr} 1}\left(\begin{array}{l}
\bar{q} \\
v
\end{array}\right), \forall(q, v) \in \mathbf{H} .
$$

Step 1: Using test functions $\phi \in \mathcal{C}_{0}^{\infty}\left(\Omega_{\mathrm{s}}\right)^{m}, \varphi \in \mathcal{C}_{0}^{\infty}\left(\Omega_{\mathrm{f}}\right)$ in 49 , all the boundary terms go to zero. Now set $\varphi=0$ (also in $(49)$ ), we obtain

$$
\int_{\Omega_{\mathrm{s}}} \boldsymbol{\sigma}: \nabla \bar{\phi} \mathrm{d} x-\omega^{2} \rho_{\mathrm{s}} \int_{\Omega_{\mathrm{s}}} u \cdot \bar{\phi} \mathrm{d} x=-\int_{\Omega_{\mathrm{s}}} A: \nabla \bar{\phi} \mathrm{d} x, \forall \boldsymbol{\phi} \in \mathcal{C}_{0}^{\infty}\left(\Omega_{\mathrm{s}}\right)^{m} .
$$

This means $-\operatorname{div} \boldsymbol{\sigma}-\omega^{2} \rho_{\mathrm{s}} u=\operatorname{div} A$ in $\left(\mathcal{C}_{0}^{\infty}\left(\Omega_{\mathrm{s}}\right)^{m}\right)^{\prime}$ i.e. in the sense of distributions. Since $u \in H^{1}\left(\Omega_{\mathrm{s}}\right)^{m}, \operatorname{div}(\boldsymbol{\sigma}+A) \in H^{1}\left(\Omega_{\mathrm{s}}\right)^{m}$. As a result, the above equality holds in $L^{2}\left(\Omega_{\mathrm{s}}\right)$,

$$
\operatorname{div}(\boldsymbol{\sigma}+A)+\omega^{2} \rho_{\mathrm{s}} u=0, \text { in } L^{2}\left(\Omega_{\mathrm{s}}\right)^{m} .
$$

Similarly, set $\phi=0$ in (49), and we obtain that $\Delta p+\kappa^{2} p=0$ in the sense of distributions. Since $p \in H^{1}\left(\Omega_{\mathrm{f}}\right), \Delta p \in H^{1}\left(\Omega_{\mathrm{f}}\right)$. As a result, we can write

$$
\Delta p+\kappa^{2} p=0, \text { in } L^{2}\left(\Omega_{\mathrm{f}}\right) .
$$

We now use Lemma 4 to make sense of the normal trace $\operatorname{div}(\boldsymbol{\sigma}+A) \cdot \nu$ and conormal trace $\frac{\partial p}{\partial \nu}$ and the integration by parts (39), (3).

Step 2: To derive the transmission and boundary conditions, we choose test functions $\phi \in \mathcal{C}^{\infty}\left(\overline{\Omega_{\mathrm{s}}}\right)^{m}, \varphi \in \mathcal{C}^{\infty}\left(\overline{\Omega_{\mathrm{f}}}\right)$. Since the arguments to obtain (44) and (43) are standard, $c f$. [12, Eqn 27-31], we will only write that for condition (42). Setting $\varphi=0$ in (49), we get

$$
\int_{\Omega_{\mathrm{s}}}(\boldsymbol{\sigma}+A): \nabla \overline{\boldsymbol{\phi}} \mathrm{d} x-\int_{\Omega_{\mathrm{s}}} \omega^{2} \rho_{\mathrm{s}} u \overline{\boldsymbol{\phi}} \mathrm{d} x+\langle p \nu, \boldsymbol{\phi}\rangle_{1 / 2, \Gamma}=0 .
$$


This is further written, by keeping $\boldsymbol{\sigma}$ and $A$ together and using IP identity (39) (given at the end of Step 1),

$$
\int_{\Omega_{\mathrm{s}}}-\operatorname{div}(\boldsymbol{\sigma}+A) \cdot \overline{\boldsymbol{\phi}} \mathrm{d} x+\langle(\boldsymbol{\sigma}+A) \cdot \nu, \boldsymbol{\phi}\rangle_{1 / 2, \Gamma}-\int_{\Omega_{\mathrm{s}}} \omega^{2} \rho_{\mathrm{s}} u \overline{\boldsymbol{\phi}} \mathrm{d} x+\langle p \nu, \boldsymbol{\phi}\rangle_{1 / 2, \Gamma}=0 .
$$

Due to 50$)$, the above identity simplifies to

$$
\langle(\boldsymbol{\sigma}+A) \cdot \nu+p \nu, \boldsymbol{\phi}\rangle_{1 / 2, \Gamma}=0, \forall \boldsymbol{\phi} \in \mathcal{C}^{\infty}\left(\overline{\Omega_{\mathrm{s}}}\right)^{m} .
$$

By density arguments, we obtain the transmission condition 442 in $H^{-1 / 2}(\Gamma)$.

Given the equivalence in the above proposition, the well-posedness of problem DP, when $\omega$ is not a Jones frequency, is obtained via that of (45). The latter follows from Theorem 2 and (46). These results are gathered in the corollary below.

Corollary 6 (Existence and Uniqueness for DP). With $\mathscr{B}$ satisfying $(14)$ and the material parameters satisfying (27), for a matrix $A \in L^{2}\left(\Omega_{s}\right)^{m \times m}$, when $\omega$ is not a Jones frequency, the problem $\mathbf{D P}(40)-(44)$ has a unique solution $(p, u) \in \mathbf{H}$. In addition, for each $(\lambda, \mu)$ and with $h$ satisfying (32), we have

$$
\begin{aligned}
& \left\|\left(\tilde{p}_{\lambda+h, \mu}, \tilde{u}_{\lambda+h, \mu}\right)\right\|_{\mathbf{H}} \leq \mathrm{d}(\lambda, \mu)\|A\|_{L^{2}\left(\Omega_{\mathrm{s}}\right)^{m \times m}}, \\
& \left\|\left(\tilde{p}_{\lambda, \mu+h}, \tilde{u}_{\lambda, \mu+h}\right)\right\|_{\mathbf{H}} \leq \mathrm{d}(\lambda, \mu)\|A\|_{L^{2}\left(\Omega_{\mathrm{s}}\right)^{m \times m}}
\end{aligned}
$$

for a constant $\mathrm{d}(\lambda, \mu)>0$ independent of $h$.

\subsection{Characterization of partial derivatives with respect to Lamé parameters}

The goal of this section is to prove that the solution to OP $15-(19)$ is separately continuous and partially Fréchet differentiable with respect to the Lamé parameters, and that their partial derivatives can be characterized as solutions to the corresponding auxiliary problem. We simplify the notation and only indicate the dependence with respect to $\lambda$ and $\mu$. In addition, we will assume that

$$
\omega \text { is not a Jones frequency. }
$$

Recall that, under this assumption, we have defined in (35) the solution operator $\mathcal{S}$ for problem $\mathbf{O P}, c f$. Corollary 3. If we set $A=\mathbf{C}^{\prime}: \boldsymbol{\epsilon}\left(u_{\lambda, \mu}\right)$ with $\mathbf{C}^{\prime} \in\left\{\partial_{\lambda} \mathbf{C}, \partial_{\mu} \mathbf{C}\right\}$ and $u_{\lambda, \mu}=\pi_{2} \mathcal{S}(\lambda, \mu)$, with $\pi_{i}$ the projection map onto the $i$-th component, then $A \in L^{2}\left(\Omega_{\mathrm{s}}\right)^{m \times m}$. A version of Corollary 6 can be obtained for this particular $A$. 
Corollary 7. Under (53) and (27), with $u_{\lambda, \mu}=\pi_{2} \mathcal{S}(\lambda, \mu)$, the problem

$$
(\mathbf{L a D P})\left\{\begin{aligned}
\operatorname{div} \boldsymbol{\sigma}+\omega^{2} \rho_{s} u & =-\operatorname{div}\left(\mathbf{C}^{\prime}: \boldsymbol{\epsilon}\left(u_{\lambda, \mu}\right)\right) & & \text { in }\left(H^{1}\left(\Omega_{s}\right)^{m}\right)^{\prime} \\
\Delta p+\kappa^{2} p & =0 & & \text { in } L^{2}\left(\Omega_{f}\right) \\
\left(\boldsymbol{\sigma}+\mathbf{C}^{\prime}: \boldsymbol{\epsilon}\left(u_{\lambda, \mu}\right)\right) \cdot \nu & =-p \nu & & \text { in } H^{-1 / 2}(\Gamma)^{m} \\
\omega^{2} \rho_{f} u \cdot \nu-\frac{\partial p}{\partial \nu} & =0 & & \text { in } H^{-1 / 2}(\Gamma) \\
\frac{\partial p}{\partial \nu} & =-\mathscr{B} p & & \text { in } H^{-1 / 2}(\Sigma)
\end{aligned}\right.
$$

has a unique solution $\left(\tilde{u}_{\lambda, \mu}, \tilde{p}_{\lambda, \mu}\right) \in \mathbf{H}$. In addition, it is equivalent to the variational problem VLaDP

$$
\begin{gathered}
\text { Find }\left(\begin{array}{c}
p \\
u
\end{array}\right) \in \mathbf{H}: \mathbf{a}\left[\left(\begin{array}{c}
p \\
u
\end{array}\right),\left(\begin{array}{c}
q \\
v
\end{array}\right)\right]=\mathbf{l}_{\mathrm{fr} 2}\left[\left(\frac{\bar{q}}{v}\right)\right], \forall\left(\begin{array}{c}
q \\
v
\end{array}\right) \in \mathbf{H} ; \\
\text { with } \mathbf{l}_{\mathrm{fr} 2} \in \mathbf{H}^{\prime}, \mathbf{l}_{\mathrm{fr} 2}\left[\left(\begin{array}{c}
q \\
v
\end{array}\right)\right] \triangleq-\int_{\Omega_{s}}\left(\mathbf{C}^{\prime}: \boldsymbol{\epsilon}\left(u_{\lambda, \mu}\right)\right): \nabla v \mathrm{~d} x .
\end{gathered}
$$

As a result, we can define the solution operators

$$
\begin{gathered}
\tilde{\mathcal{S}}_{\lambda}, \tilde{\mathcal{S}}_{\mu}: \mathbb{R}^{+} \times \mathbb{R}^{+} \rightarrow \mathbf{H} \quad, \quad(\lambda, \mu) \longmapsto\left(\tilde{p}_{\lambda, \mu}, \tilde{u}_{\lambda, \mu}\right), \\
\text { with }\left(\tilde{p}_{\lambda, \mu}, \tilde{u}_{\lambda, \mu}\right) \text { the unique solution in } \mathbf{H} \text { to }(54)-(58) \text { for } \\
\mathbf{C}^{\prime}=\partial_{\lambda} \mathbf{C} \text { and } \mathbf{C}^{\prime}=\partial_{\mu} \mathbf{C} \text { respectively }
\end{gathered}
$$

Proposition 8. Under assumption (53), the mappings $\lambda \mapsto \mathcal{S}(\lambda, \mu)$ and $\mu \mapsto$ $\mathcal{S}(\lambda, \mu)$ are continuous and Fréchet differentiable. Their derivatives are unique solutions to (54)-(58). In particular,

$$
\partial_{\lambda} \mathcal{S}(\lambda, \mu)=\tilde{\mathcal{S}}_{\lambda}(\lambda, \mu) \quad, \quad \partial_{\mu} \mathcal{S}(\lambda, \mu)=\tilde{\mathcal{S}}_{\mu}(\lambda, \mu) .
$$

Proof. Since the proof for $\mu$ is identical, only that for $\lambda$ is written. Since $\mathbf{C}_{\lambda}$ is separately affine in $\lambda$ and $\mu$, we have

$$
\mathbf{C}_{\lambda+h}-\mathbf{C}_{\lambda}=\mathbf{C}_{\lambda}^{\prime} h \text { with } \mathbf{C}_{\lambda} \triangleq \mathbf{C}(\lambda, \mu), \mathbf{C}_{\lambda}^{\prime} \triangleq\left(\partial_{\lambda} \mathbf{C}\right)(\lambda, \mu) .
$$

For a fixed $\mu$, consider the unique solutions to $\mathrm{OP}$ at $\lambda+h$ and $\lambda$, and that to LaDP at $\lambda$,

$$
\left(p_{\lambda+h}, u_{\lambda+h}\right)=\mathcal{S}(\lambda+h, \mu) ;\left(p_{\lambda}, u_{\lambda}\right)=\mathcal{S}(\lambda, \mu) ;\left(\tilde{p}_{\lambda}, \tilde{u}_{\lambda}\right)=\tilde{\mathcal{S}}_{\lambda}(\lambda, \mu) .
$$

These quantities have the following regularity,

$$
\begin{gathered}
\left(\tilde{p}_{\lambda}, \tilde{u}_{\lambda}\right),\left(p_{\lambda+h}, u_{\lambda+h}\right),\left(p_{\lambda}, u_{\lambda}\right) \in H^{1}\left(\Omega_{\mathrm{f}}\right) \times H^{1}\left(\Omega_{\mathrm{s}}\right)^{m} \\
\Delta p_{\lambda+h}, \Delta p_{\lambda}, \Delta \tilde{p}, \Delta \tilde{p}_{\lambda} \in L^{2}\left(\Omega_{\mathrm{f}}\right) \\
\operatorname{div}\left(\boldsymbol{\sigma}(\tilde{u})+\mathbf{C}_{\lambda}^{\prime}: \boldsymbol{\epsilon}\left(u_{\lambda}\right)\right) \in L^{2}\left(\Omega_{\mathrm{s}}\right)^{m} c f . \text { Lemma } 4 .
\end{gathered}
$$


We will show that their difference $\left(p_{\lambda+h}-p_{\lambda}, u_{\lambda+h}-u_{\lambda}\right)$, and $\left(\hat{p}_{\lambda}, \hat{u}_{\lambda}\right) \in \mathbf{H}$ with

$$
\hat{u}_{\lambda} \triangleq u_{\lambda+h}-u_{\lambda}-h \tilde{u}_{\lambda} \quad, \quad \hat{p}_{\lambda} \triangleq p_{\lambda+h}-p_{\lambda}-h \tilde{p}_{\lambda}
$$

satisfy,

$$
\left\|\left(p_{\lambda+h}-p_{\lambda}, u_{\lambda+h}-u_{\lambda}\right)\right\|_{\mathbf{H}}=\mathrm{O}(|h|),\left\|\left(\hat{p}_{\lambda}, \hat{u}_{\lambda}\right)\right\|_{\mathbf{H}}=\mathrm{o}(|h|), h \rightarrow 0 .
$$

Step 1a: Consider the volume identity in $\Omega_{\mathrm{s}}$ of each system,

$$
\begin{aligned}
\operatorname{div}\left(\mathbf{C}_{\lambda+h}: \boldsymbol{\epsilon}\left(u_{\lambda+h}\right)\right)+\omega^{2} \rho_{\mathrm{s}} u_{\lambda+h}=0 & \text { in } L^{2}\left(\Omega_{\mathrm{s}}\right)^{m} \\
-\operatorname{div}\left(\mathbf{C}_{\lambda}: \boldsymbol{\epsilon}\left(u_{\lambda}\right)\right)-\omega^{2} \rho_{\mathrm{s}} u_{\lambda}=0 & \text { in } L^{2}\left(\Omega_{\mathrm{s}}\right)^{m} \\
-h\left(\operatorname{div}\left(\mathbf{C}_{\lambda}: \boldsymbol{\epsilon}\left(\tilde{u}_{\lambda}\right)\right)+\omega^{2} \rho_{\mathrm{s}} \tilde{u}_{\lambda}+\operatorname{div} \mathbf{C}_{\lambda}^{\prime}: \boldsymbol{\epsilon}\left(u_{\lambda}\right)\right)=0 & \text { in }\left(H^{1}\left(\Omega_{\mathrm{s}}\right)^{m}\right)^{\prime} .
\end{aligned}
$$

In order to rewrite the above expressions, we use 'as a buffer'

$$
-\operatorname{div} \mathbf{C}_{\lambda}: \boldsymbol{\epsilon}\left(u_{\lambda+h}\right)+\operatorname{div} \mathbf{C}_{\lambda}: \boldsymbol{\epsilon}\left(u_{\lambda+h}\right)=0 \quad \text { in }\left(H^{1}\left(\Omega_{\mathrm{s}}\right)^{m}\right)^{\prime} .
$$

Adding the above four equations $(64)+(65)+(66)+(67)$, replacing $\mathbf{C}_{\lambda+h}-\mathbf{C}_{\lambda}$ with $\mathbf{C}_{\lambda}^{\prime} h$, and after some rearrangement, we obtain

$$
\begin{gathered}
\operatorname{div} \mathbf{C}_{\lambda}: \boldsymbol{\epsilon}\left(\hat{u}_{\lambda}\right)+\omega^{2} \rho_{\mathrm{s}} \hat{u}_{\lambda}=-\operatorname{div} \mathrm{E} \quad \text { in }\left(H^{1}\left(\Omega_{\mathrm{s}}\right)^{m}\right)^{\prime} ; \\
\text { with } \mathbf{E} \triangleq h \mathbf{C}_{\lambda}^{\prime}: \boldsymbol{\epsilon}\left(u_{\lambda+h}-u_{\lambda}\right) \quad \text { in } L^{2}\left(\Omega_{\mathrm{s}}\right)^{m \times m} .
\end{gathered}
$$

Since $\hat{u}_{\lambda} \in H^{1}\left(\Omega_{\mathrm{s}}\right)$ and $\mathrm{E} \in L^{2}\left(\Omega_{\mathrm{s}}\right)^{m \times m}$, by Lemma 4 , the following normal trace is well-defined,

$$
\left(\mathbf{C}_{\lambda}: \boldsymbol{\epsilon}\left(\hat{u}_{\lambda}\right)+\mathrm{E}\right) \cdot \nu \in H^{-1 / 2}(\Gamma)^{m} .
$$

Working in the same manner with $(64)+65)+67$, also replacing $\mathbf{C}_{\lambda+h}-\mathbf{C}_{\lambda}$, we obtain an equation for $\left(p_{\lambda+h}-p_{\lambda}, u_{\lambda+h}-u_{\lambda}\right)$,

$$
\begin{gathered}
\operatorname{div} \mathbf{C}_{\lambda}: \boldsymbol{\epsilon}\left(u_{\lambda+h}-u_{\lambda}\right)+\omega^{2} \rho_{\mathrm{s}}\left(u_{\lambda+h}-u_{\lambda}\right)=-\operatorname{dive} \quad \text { in }\left(H^{1}\left(\Omega_{\mathrm{s}}\right)^{m}\right)^{\prime} ; \\
\text { with } \mathrm{e} \triangleq h \mathbf{C}_{\lambda}^{\prime}: \boldsymbol{\epsilon}\left(u_{\lambda+h}\right) \quad \text { in } L^{2}\left(\Omega_{\mathrm{S}}\right)^{m \times m} .
\end{gathered}
$$

Similarly, since $u_{\lambda+h}-u_{\lambda} \in H^{1}\left(\Omega_{\mathrm{s}}\right)$ and e $\in L^{2}\left(\Omega_{\mathrm{s}}\right)^{m \times m}$, Lemma 4 gives

$$
\left(\mathbf{C}_{\lambda}: \boldsymbol{\epsilon}\left(u_{\lambda+h}-u_{\lambda}\right)+\mathrm{e}\right) \cdot \nu \in H^{-1 / 2}(\Gamma)^{m} .
$$

Step 1b: We next work with the kinematic interface condition in each problem,

$$
\begin{aligned}
\left(\mathbf{C}_{\lambda+h}: \boldsymbol{\epsilon}\left(u_{\lambda+h}\right)\right) \cdot \nu+p_{\lambda+h} \nu & =-p_{\text {inc }} \nu \quad \text { in } H^{-1 / 2}(\Gamma)^{m} \\
-\left(\mathbf{C}_{\lambda}: \boldsymbol{\epsilon}\left(u_{\lambda}\right)\right) \cdot \nu-p_{\lambda} \nu & =p_{\text {inc }} \nu \quad \text { in } H^{-1 / 2}(\Gamma)^{m} \\
-h\left(\mathbf{C}_{\lambda}: \boldsymbol{\epsilon}(\tilde{u})+\mathbf{C}_{\lambda}^{\prime}: \boldsymbol{\epsilon}\left(u_{\lambda}\right)\right) \cdot \nu-h \tilde{p} \nu & =0 \quad \text { in } H^{-1 / 2}(\Gamma)^{m} .
\end{aligned}
$$


A 'buffer' in the form of normal trace is needed as before. However, to maintain the current regularity (in $H^{-1 / 2}(\Gamma)^{m}$ ), thanks to (69), we can use

$$
-\left(\mathbf{C}_{\lambda}: \boldsymbol{\epsilon}\left(\hat{u}_{\lambda}\right)+\mathrm{E}\right) \cdot \nu+\left(\mathbf{C}_{\lambda}: \boldsymbol{\epsilon}\left(\hat{u}_{\lambda}\right)+\mathrm{E}\right) \cdot \nu=0 \text { in } H^{-1 / 2}(\Gamma)^{m} .
$$

When adding the above four equations together, the sum of all first terms simplifies to zerd 12 , we obtain the kinematic interface condition,

$$
\left(\mathbf{C}_{\lambda}: \boldsymbol{\epsilon}\left(\hat{u}_{\lambda}\right)+\mathbf{E}\right) \cdot \nu+\hat{p}_{\lambda} \nu=0 \text { in } H^{-1 / 2}(\Gamma)^{m} .
$$

To derive the interface condition for $u_{\lambda+h}-u_{\lambda}$, we use the same idea as before to maintain regularity in $H^{-1 / 2}(\Gamma)^{m}$. Using (71), we add (72) and (73) to

$$
-\left(\mathbf{C}_{\lambda}: \boldsymbol{\epsilon}\left(u_{\lambda+h}-u_{\lambda}\right)+\mathrm{e}\right) \cdot \nu+\left(\mathbf{C}_{\lambda}: \boldsymbol{\epsilon}\left(u_{\lambda+h}-u_{\lambda}\right)+\mathrm{e}\right) \cdot \nu=0 \in H^{-1 / 2}(\Gamma)^{m} .
$$

After some rearrangement and simplification, we find

$$
\left(\mathbf{C}_{\lambda}: \boldsymbol{\epsilon}\left(u_{\lambda+h}-u_{\lambda}\right)+\mathrm{e}\right) \cdot \nu+\left(p_{\lambda+h}-p_{\lambda}\right) \nu=0 \quad \text { in } H^{-1 / 2}(\Gamma)^{m} .
$$

Step 1c: We next work with the Helmholtz equation, kinematic transmission condition (18), and ABC in each problem. Taking

$$
\left.((16),(18),(19))\right|_{\lambda+h}-\left.((16),(18),(19))\right|_{\lambda}-\left.h((55),(57),(58))\right|_{\lambda},
$$

we obtain

$$
\begin{aligned}
& \Delta \hat{p}_{\lambda}+\kappa^{2} \hat{p}_{\lambda}=0 \in L^{2}\left(\Omega_{\mathrm{f}}\right) ; \\
& \omega^{2} \rho_{\mathrm{f}} \hat{u}_{\lambda} \cdot \nu=\frac{\partial \hat{p}_{\lambda}}{\partial \nu} \in H^{-1 / 2}(\Gamma) ; \frac{\partial \hat{p}_{\lambda}}{\partial \nu}=\mathscr{B} \hat{p}_{\lambda} \in H^{-1 / 2}(\Sigma) .
\end{aligned}
$$

Using $\left.((16),(18),(19))\right|_{\lambda+h}-\left.((16),(18),(19))\right|_{\lambda}$, we obtain the exact same system as above with $\hat{u}_{\lambda}$ replaced by $u_{\lambda+h}-u_{\lambda}$ and $\hat{p}_{\lambda}$ replaced by $p_{\lambda+h}-p_{\lambda}$.

At the end of Step $1 \mathrm{a}-1 \mathrm{c}$, we have obtained $\mathrm{E} \in L^{2}\left(\Omega_{\mathrm{s}}\right)^{m \times m}$ and $\left(\hat{p}_{\lambda}, \hat{u}_{\lambda}\right) \in \mathbf{H}$ satisfying (40)-(44) with $A$ replaced by E. Similarly, we have e $\in L^{2}\left(\Omega_{\mathrm{s}}\right)^{m \times m}$ and $\left(p_{\lambda+h}-p_{\lambda}, u_{\lambda+h}-u_{\lambda}\right) \in \mathbf{H}$ satisfying (40) with $A$ replaced by e.

${ }^{12}$ We first group the first terms of 74 and $(75)$, and use the definition of $\mathrm{E}$,

$$
\left(-\mathbf{C}_{\lambda}: \boldsymbol{\epsilon}(h \tilde{u})-h \mathbf{C}_{\lambda}^{\prime}: \boldsymbol{\epsilon}\left(u_{\lambda}\right)-\mathbf{C}_{\lambda}: \boldsymbol{\epsilon}\left(\hat{u}_{\lambda}\right)-h \mathbf{C}_{\lambda}^{\prime}: \boldsymbol{\epsilon}\left(u_{\lambda+h}-u_{\lambda}\right)\right) \cdot \nu .
$$

With the last three terms together grouped together, the above expression simplifies to $\left(-\mathbf{C}_{\lambda}: \boldsymbol{\epsilon}(h \tilde{u})+\mathbf{C}_{\lambda}: \boldsymbol{\epsilon}\left(u_{\lambda}+h \tilde{u}_{\lambda}\right)-\mathbf{C}_{\lambda+h}: \boldsymbol{\epsilon}\left(u_{\lambda+h}\right)\right) \cdot \nu=\left(\mathbf{C}_{\lambda}: \boldsymbol{\epsilon}\left(u_{\lambda}\right)-\mathbf{C}_{\lambda+h}: \boldsymbol{\epsilon}\left(u_{\lambda+h}\right)\right) \cdot \nu$.

Now adding the first terms of $(72$ and $(73)$, we get zero. Note that all the above expressions are in $H^{-1 / 2}(\Gamma)^{m}$. 
Step 2: We next bound the differences. For the rest of the discussion, we assume that $h$ satisfies (32). From Corollary 6, under assumption (53), we have existence and uniqueness of solutions, and the energy estimate 52 gives

$$
\begin{aligned}
\left\|\left(p_{\lambda+h}-p_{\lambda}, u_{\lambda+h}-u_{\lambda}\right)\right\|_{\mathbf{H}} & \leq \mathrm{d}(\lambda, \mu)\|\mathrm{e}\|_{L^{2}\left(\Omega_{\mathrm{s}}\right)^{m \times m}} \\
\left\|\left(\hat{p}_{\lambda}, \hat{u}_{\lambda}\right)\right\|_{\mathbf{H}} & \leq \mathrm{d}(\lambda, \mu)\|\mathrm{E}\|_{L^{2}\left(\Omega_{\mathrm{s}}\right)^{m \times m}} .
\end{aligned}
$$

Using its definition, we can bound e by

$$
\begin{aligned}
\|\mathrm{e}\|_{L^{2}\left(\Omega_{\mathrm{s}}\right)^{m \times m}} & \leq\left\|\mathbf{C}_{\lambda+h}-\mathbf{C}_{\lambda}\right\|_{\infty}\left\|\boldsymbol{\epsilon}\left(u_{\lambda+h}\right)\right\|_{L^{2}\left(\Omega_{\mathrm{s}}\right)^{m \times m}} \\
& \stackrel{34}{\leq}|h|\left\|\mathbf{C}_{\lambda}^{\prime}\right\|_{\infty} \mathrm{c}(\lambda, \mu)\left\|p_{\mathrm{inc}}\right\|_{H^{2}\left(\Omega_{\mathrm{f}}\right)} .
\end{aligned}
$$

With $\mathbf{C}_{\lambda}^{\prime}$ being constant, there exists $\tilde{\mathrm{c}}(\lambda, \mu)>0$ independent of $h$ such that

$$
\left\|\left(p_{\lambda+h}-p_{\lambda}, u_{\lambda+h}-u_{\lambda}\right)\right\|_{\mathbf{H}} \leq|h| \tilde{\mathrm{c}}(\lambda, \mu)\left\|p_{\text {inc }}\right\|_{H^{2}\left(\Omega_{\mathrm{f}}\right)} .
$$

From this follows the continuity of the mapping $\lambda \mapsto \mathcal{S}(\lambda, \mu)$.

Similarly, the bound of $\mathrm{E}$ follows from its definition,

$\|\mathrm{E}\|_{L^{2}\left(\Omega_{\mathrm{s}}\right)^{m \times m}}=\left\|h \mathbf{C}_{\lambda}^{\prime}: \boldsymbol{\epsilon}\left(u_{\lambda+h}-u_{\lambda}\right)\right\|_{L^{2}\left(\Omega_{\mathrm{s}}\right)^{m \times m}} \leq|h|\left\|\mathbf{C}_{\lambda}^{\prime}\right\|_{\infty}\left\|u_{\lambda+h}-u_{\lambda}\right\|_{H^{1}\left(\Omega_{\mathrm{s}}\right)^{m}}$.

Combining with 82 , we obtain a constant $\tilde{\mathrm{d}}(\lambda, \mu)>0$ independent of $h$ such that

$$
\left\|\left(\hat{p}_{\lambda}, \hat{u}_{\lambda}\right)\right\|_{\mathbf{H}} \leq|h|^{2} \tilde{\mathrm{d}}(\lambda, \mu)\left\|p_{\text {inc }}\right\|_{H^{2}\left(\Omega_{\mathrm{f}}\right)} .
$$

As a result, $\left\|\hat{p}_{\lambda}, \hat{u}_{\lambda}\right\|_{\mathbf{H}}=\mathrm{o}(h)$. This finishes the proof that $\lambda \mapsto \mathcal{S}$ is Fréchet differentiable with derivative $\partial_{\lambda} \mathcal{S}=\tilde{\mathcal{S}}_{\lambda}(\lambda, \mu)$.

Remark 6. A more natural buffer than that employed in 75 would have been the 'formal' normal trace of $-\mathbf{C}_{\lambda}: \boldsymbol{\epsilon}\left(u_{\lambda+h}\right) \in\left(H^{1}\left(\Omega_{\mathrm{s}}\right)^{m}\right)^{\prime}$, which is the buffer in (67). However, under Lipshitz assumption, this 'formal' normal trace does not make sense in $H^{s}(\Gamma),|s| \leq 1$. That is why we work with the buffer terms given in (69) and 71), which have well-defined normal trace.

Differentiability of approximate far-field-pattern (a-FFP) under assumption (53). We recall that the a-FFP operator $\mathrm{F}(\lambda, \mu)$ associated to $\mathrm{OP}$ is given in (37). The a-FFP operator associated to LaDP can be defined in a similar manner,

$$
\begin{gathered}
\tilde{\mathrm{F}}_{\bullet}: \mathbb{R}^{+} \times \mathbb{R}^{+} \longrightarrow \mathcal{C}^{\infty}\left(\mathbb{S}^{1}, \mathbb{C}\right) \quad, \quad(\lambda, \mu) \longrightarrow \tilde{\mathrm{F}}_{\bullet}(\lambda, \mu) \\
\left(\tilde{\mathrm{F}} \bullet(\lambda)(\hat{x})=\mathfrak{c}(m) \int_{\Gamma}\left(\Phi(\hat{x}, y) \tilde{p}(y)-\omega^{2} \rho_{\mathrm{f}} \Psi(\hat{x}, y) \tilde{u}(y) \cdot \nu\right) \mathrm{d} s(y) .\right. \\
\text { where } \quad(\tilde{p}, \tilde{u})=\tilde{\mathcal{S}}_{\bullet}(\lambda, \mu) \quad, \quad \text { for } \bullet=\lambda, \mu .
\end{gathered}
$$


Here, we have written

$$
\Phi(\hat{x}, y) \triangleq \frac{\partial e^{-\mathrm{i} \kappa \hat{x} \cdot y}}{\partial \nu(y)} \quad ; \quad \Psi(\hat{x}, y) \triangleq e^{-\mathrm{i} \kappa \hat{x} \cdot y} .
$$

We proceed to show their separate continuity and differentiability.

Proposition 9. The mappings $\lambda \mapsto \mathrm{F}(\lambda, \mu)$ and $\mu \mapsto \mathrm{F}(\lambda, \mu)$ are continuous and Fréchet differentiable with

$$
\partial_{\lambda} \mathrm{F}(\lambda, \mu)=\tilde{\mathrm{F}}_{\lambda}(\lambda, \mu) \quad, \quad \partial_{\mu} \mathrm{F}(\lambda, \mu)=\tilde{\mathrm{F}}_{\mu}(\lambda, \mu) .
$$

Proof. We will only write out the proof with respect to $\lambda$, that for $\mu$ is completely similar. We will show that

$$
\mathrm{F}(\lambda+h)-\mathrm{F}(\lambda)=\mathrm{O}(|h|), \mathrm{F}(\lambda+h)-\mathrm{F}(\lambda)-h \tilde{\mathrm{F}}(\lambda)=\mathrm{o}(|h|), h \rightarrow 0 .
$$

In terms of the quantities defined in 61 -63), the differences of the a-FFPs are

$$
\begin{gathered}
\mathrm{F}(\lambda+h)-\mathrm{F}(\lambda)-h \tilde{\mathrm{F}}(\lambda)=\mathfrak{c}(m) \int_{\Gamma}\left(\Phi(\hat{x}, y) \hat{p}_{\lambda}(y)-\omega^{2} \rho_{\mathrm{f}} \Psi(\hat{x}, y) \hat{u}_{\lambda}(y) \cdot \nu\right) \mathrm{d} s(y) \\
\mathrm{F}(\lambda+h)-\mathrm{F}(\lambda)=\mathfrak{c}(m) \int_{\Gamma}\left(\Phi(\hat{x}, y)\left(p_{\lambda+h}-p_{\lambda}\right)(y)-\right. \\
\left.\omega^{2} \rho_{\mathrm{f}} \Psi(\hat{x}, y)\left(u_{\lambda+h}-u_{\lambda}\right)(y) \cdot \nu\right) \mathrm{d} s(y) .
\end{gathered}
$$

Using $|\Phi(\hat{x}, y)|=1$ and $|\Psi(\hat{x}, y)|=|\mathrm{i} \kappa \hat{x} \cdot \hat{n}|=\kappa$, we obtain a constant $\mathrm{c}=$ $\mathrm{c}\left(n, \Gamma, \omega, \rho_{\mathrm{f}}, \kappa\right)>0$ independent of $h$ so that

$$
|\mathrm{F}(\lambda+h)-\mathrm{F}(\lambda)-h \tilde{\mathrm{F}}(\lambda)| \leq \mathrm{c}\left(\left\|\hat{p}_{\lambda}\right\|_{L^{2}(\Gamma)}+\left\|\hat{u}_{\lambda}\right\|_{L^{2}(\Gamma)}\right) \leq \mathrm{c}\left\|\left(\hat{p}_{\lambda}, \hat{u}_{\lambda}\right)\right\|_{\mathbf{H}} .
$$

Similarly, there exists a constant $\tilde{\mathrm{c}}=\tilde{\mathrm{c}}\left(n, \Gamma, \omega, \rho_{\mathrm{f}}, \kappa\right)>0$ independent of $h$ so that

$$
|\mathrm{F}(\lambda+h)-\mathrm{F}(\lambda)| \leq \tilde{\mathrm{c}}\left\|\left(p_{\lambda+h}-p_{\lambda}, u_{\lambda+h}-u_{\lambda}\right)\right\|_{\mathbf{H}} .
$$

Separate continuity is obtained by using the bound (82) for $\left\|p_{\lambda+h}-p_{\lambda}, u_{\lambda+h}-u_{\lambda}\right\|_{\mathbf{H}}$, and partial Fréchet differentiability by (83) for $\left\|\left(\hat{p}_{\lambda}, \hat{u}_{\lambda}\right)\right\|_{\mathbf{H}}$.

\subsection{Characterization of the partial derivatives with respect to solid density}

The analysis with respect to the solid density $\rho_{\mathrm{s}}$ is simpler. The corresponding auxiliary problem has better regularity; it has no interface source terms and, instead, only a very regular volume one (in $\left.H^{1}\left(\Omega_{\mathrm{s}}\right)^{m}\right)$. As a result, its PDE form is readily well-defined in standard Sobolev spaces. For the current discussion, the 
notation is simplified to indicate only the dependence with respect to $\rho_{\mathrm{s}}$. With $u_{\rho_{\mathrm{s}}}=\mathcal{S}\left(\rho_{\mathrm{s}}\right)=\mathcal{S}\left(\lambda, \mu, \rho_{\mathrm{s}}\right) c f$. 35), the auxiliary problem with respect to $\rho_{\mathrm{s}}$ is

$$
(\text { SoDP })\left\{\begin{aligned}
\operatorname{div} \boldsymbol{\sigma}+\omega^{2} \rho_{\mathrm{s}} u & =-\omega^{2} u_{\rho_{\mathrm{s}}} & & \text { in } L^{2}\left(\Omega_{\mathrm{s}}\right)^{m} \\
\Delta p+\kappa^{2} p & =0 & & \text { in } L^{2}\left(\Omega_{\mathrm{f}}\right) \\
\boldsymbol{\sigma} \cdot \nu & =-p \nu & & \text { in } H^{-1 / 2}(\Gamma)^{m} \\
\omega^{2} \rho_{\mathrm{f}} u \cdot \nu-\frac{\partial p}{\partial \nu} & =0 & & \text { in } H^{-1 / 2}(\Gamma) \\
\frac{\partial p}{\partial \nu} & =-\mathscr{B} p & & \text { in } H^{-1 / 2}(\Sigma) .
\end{aligned}\right.
$$

The arguments to obtain the variational formulation equivalent to SoDP and the well-posedness for both problems are standard. The latter follows from Theorem 2.

Proposition 10. Problem SoDP is equivalent to variational problem

$$
\begin{gathered}
\text { Find }\left(\begin{array}{c}
p \\
u
\end{array}\right) \in \mathbf{H}: \mathbf{a}\left[\left(\begin{array}{c}
p \\
u
\end{array}\right),\left(\begin{array}{c}
q \\
v
\end{array}\right)\right]=\mathbf{l}_{\mathrm{fr} 3}\left[\left(\begin{array}{c}
\bar{q} \\
v
\end{array}\right)\right], \forall\left(\begin{array}{c}
q \\
v
\end{array}\right) \in \mathbf{H} ; \\
\text { with } \mathbf{l}_{\mathrm{fr} 3} \in \mathbf{H}^{\prime}, \mathbf{1}_{\mathrm{fr} 3}\left[\left(\begin{array}{c}
q \\
v
\end{array}\right)\right] \triangleq-\int_{\Omega_{s}} \omega^{2} u_{\rho_{\mathrm{s}}}: v \mathrm{~d} x .
\end{gathered}
$$

Given (53), there exist unique solutions to both problems in $\mathbf{H}$.

As a result of this, we can define the solution operator:

$$
\begin{gathered}
\tilde{\mathcal{S}}: \mathbb{R}^{+} \rightarrow \mathbf{H} \quad, \quad \rho_{\mathrm{s}} \longmapsto \tilde{\mathcal{S}}\left(\rho_{\mathrm{s}}\right) \\
\text { with } \tilde{\mathcal{S}}\left(\rho_{\mathrm{s}}\right) \text { the unique solution in } \mathbf{H} \text { to } 86-90 .
\end{gathered}
$$

The a-FFP associated to SoDP, denoted by $\tilde{\mathrm{F}}\left(\rho_{\mathrm{s}}\right)$, is defined using (84) with $(\tilde{p}, \tilde{u})=\tilde{S}\left(\rho_{\mathrm{s}}\right)$.

The proofs for continuity and partial differentiability in this case are simpler than those for the Lamé parameters. In particular, we do not encounter the problem discussed in Remark 6. These results are gathered in the following proposition.

Proposition 11. Given (53), at fixed $(\lambda, \mu)$, the mapping $\rho_{s} \mapsto \mathcal{S}\left(\rho_{\mathrm{s}}\right)$ is continuous and Fréchet differentiable with derivative given as the unique solution to (86)(90). In particular, $\partial_{\rho_{\mathrm{s}}} \mathcal{S}\left(\rho_{\mathrm{s}}\right)=\tilde{\mathcal{S}}\left(\rho_{\mathrm{s}}\right)$. Regarding a-FFP, the mapping $\rho_{\mathrm{s}} \mapsto \tilde{\mathrm{F}}\left(\rho_{\mathrm{s}}\right)$ (defined in (37)) is continuous and Fréchet differentiable with $\partial_{\rho_{\mathrm{s}}} \mathrm{F}\left(\rho_{\mathrm{s}}\right)=\tilde{\mathrm{F}}\left(\rho_{\mathrm{s}}\right)$.

\section{Numerical validation}

In this section, to evaluate the accuracy of this characterization, we consider a $2 \mathrm{D}$ setting with circular geometries, in which there are analytical expressions for 


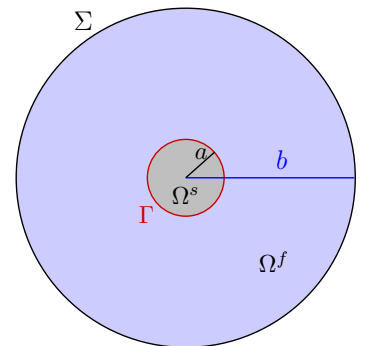

(a) Computational domain $a=1 \mathrm{~cm}, b=7.5 \mathrm{~cm}$.

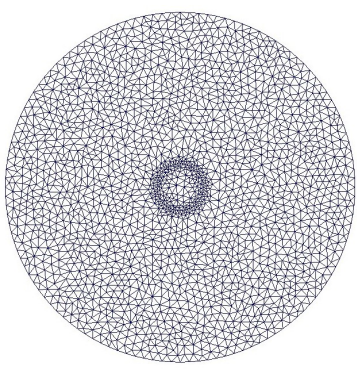

(b) Meshed domain at $f=71.61 \mathrm{kHz}(\kappa a=3)$.

Figure 2: Circular computational domain $\Omega_{\text {finite }}$ with circular obstacle $\Omega_{\mathrm{s}}$. The mesh is used with IPDG method and satisfies 7 points per wavelength, $c f$. Remark 7. Boundary $\Sigma$ and interface $\Lambda$ are discretized using curved-edge elements $c f$. [13, Sec. 3.1].

the displacement fields, a-FFP and their derivatives. This allows for a comparison between analytical values and numerical ones. The latter are obtained by using IPDG method equipped with curved-boundary edge elements, $c f$. [13, to discretize the variational problems (VOP and VLaDP).

Parameters for experiments. We follow [38, 13] and consider a setup in which a time-harmonic planewave propagating along $x$-axis is diffracted by an infinitely long steel cylinder immersed in water. Here, the coordinates of $\mathbb{R}^{3}$ are written as $(x, y, z)$ and of $\mathbb{R}^{2}$ as $(x, y)$. The cylinder has radius $a=1 \mathrm{~cm}$ and principal axis parallel to $z$-direction. The properties of the involved materials are summarized in Table 1. This $3 \mathrm{D}$ phenomenon can be approximated by a $2 \mathrm{D}$ problem, in which the solid region $\Omega_{\mathrm{s}}$ represents the circular cross-section of the cylinder in $(x, y)$-plane. The computation domain $\Omega_{\text {finite }}$ is chosen to be a disc of radius $b=7.5 \mathrm{~cm}$, see Figure 2(a), and the first-order $\mathrm{ABC}$ with $\mathscr{B}=\mathbf{T}_{1}$ defined in 20 is used.

\begin{tabular}{|c|c|c|c|c|c|}
\hline \multirow{2}{*}{ Medium } & $\begin{array}{c}\text { Density } \\
\left(\mathrm{kg} \mathrm{m}^{-3}\right)\end{array}$ & \multicolumn{2}{|c|}{$\begin{array}{c}\text { Propagation speed } \\
\left(\mathrm{m} \mathrm{s}^{-1}\right)\end{array}$} & \multicolumn{2}{|c|}{$\begin{array}{c}\text { Lamé parameters } \\
(\mathrm{GPa})\end{array}$} \\
\cline { 2 - 6 } & $\rho$ & $\mathrm{c}_{\mathrm{P}}$ & $\mathrm{c}_{\mathrm{S}}$ & $\lambda$ & $\mu$ \\
\hline Water & 1000 & 1500 & $\mathrm{n} / \mathrm{a}$ & 2.25 & $\mathrm{n} / \mathrm{a}$ \\
Steel & 7900 & 5837 & 3100 & 115.40 & 76.9 \\
\hline
\end{tabular}

Table 1: Properties of fluid and solid medium in the expriments.

In $(x, y)$ - and polar coordinates, the spatial part of a time-harmonic planewave of frequency $f=\frac{\omega}{2 \pi}$, is

$$
p_{\mathrm{pw}}(x, y)=e^{\mathrm{i} \frac{\omega}{\mathrm{c}_{\mathrm{f}}}(x, y) \cdot(1,0)}=p_{\mathrm{pw}}(r, \theta)=e^{\mathrm{i} \frac{\omega}{\mathrm{c}_{\mathrm{f}}} r \cos \theta} .
$$


Experiments are carried out for frequency $f$ and normalized wavenumber $\kappa a$ range

$$
23 \mathrm{kHz} \leq f \leq 477 \mathrm{kHz} \quad \Leftrightarrow \quad 1 \leq \kappa a \leq 20
$$

We compare between the IPDG and analytical values of the quantities listed in Table 2. To quantify the difference between an analytical value $g$ and the corresponding value $g_{h}$ computed with IPDG, we use $L^{2}$-relative error,

$$
\mathfrak{e}(g)=\frac{\left\|g-g_{h}\right\|_{2}}{\|g\|_{2}} \times 100 .
$$

\begin{tabular}{|c|c|c|c|c|c|c|}
\hline & Fluid pressure & Solid displacement & a-FFP & \multicolumn{3}{|c|}{ Fréchet derivatives } \\
\hline IPDG & $p_{h}$ & $u_{h}=\left(\left(u_{h}\right)_{x},\left(u_{h}\right)_{y}\right)$ & $\left(p_{h}\right)_{a, \infty}$ & $\partial p_{h}$ & $\partial u_{\mathrm{h}}$ & $\partial\left(p_{h}\right)_{a, \infty}$ \\
\hline Exact & $p$ & $u=\left(u_{x}, u_{y}\right)$ & $p_{a, \infty}$ & $\partial p$ & $\partial u$ & $\partial p_{a, \infty}$ \\
\hline
\end{tabular}

Table 2: Compared quantities. For the Fréchet derivatives, we write $\partial$ to mean either $\partial_{\lambda}$ or $\partial_{\mu}$. Subscript $x$ or $y$ indicates corresponding components of the solid displacement.

Method of calculation. To calculate $\left(p_{h}, u_{h}\right)$, variational problem VOP (33) is discretized by IPDG of order $\mathbb{P}_{5}$ with curved-boundary edges also of order $\mathbb{P}_{5}$, implemented in the software Hou10ni $\left[3\right.$. The displacement $u_{h}$ is then used in VLaDP (59), also discretized by IPDG to give $\left(\partial p_{h}, \partial u_{h}\right)$. We use the same interior penalty terms as in [13, Eqn 4-5]. Note that our sesquilinear form differs from [13] in the ABC. Since our goal is not to study convergence order, parameters are chosen to obtain the best precision associated with $\mathbb{P}_{5}$. In particular, the mesh is changed at each different frequency according to the criteria described in Remark 7.

Remark 7 (Mesh criteria). In all of our experiments, at each frequency $f$, the mesh, cf. Figure 2(b), is created by the software triangl $e^{14}$ taking three variables: the number of discretization points on interface $\Gamma$ and boundary $\Sigma$, denoted by $N_{\Gamma}$ and $N_{\Sigma}$ respectively, and elemental area $\mathfrak{a}_{h}$. These are defined by

$$
N_{\Gamma} \triangleq\left[\frac{2 \pi a}{h}\right] \quad, \quad N_{\Sigma} \triangleq\left[\frac{2 \pi b}{h}\right] \quad, \quad \mathfrak{a}_{h} \triangleq \frac{h^{2}}{2},
$$

in terms of 'mesh size' $h$. The quantity 'mesh size' $h$ defined as

$$
h \triangleq \frac{\lambda_{\mathrm{f}}}{N_{\mathrm{ppwl}}}=\frac{2 \pi \mathrm{c}_{\mathrm{f}}}{\omega N_{\mathrm{ppwl}}},
$$

\footnotetext{
${ }^{13}$ Degree $\mathbb{P}_{5}$ corresponds to 21 degree of freedoms per mesh element (triangle). Triangles with curved-boundary edges are used at the fluid-solid interface, cf. [13, Figure 1]. For Hou10ni, see team.inria.fr/magique3d/software/hou10ni/.

${ }^{14}$ https ://www.cs.cmu.edu/ quake/triangle.html
} 
is controlled by the frequency $f$, and the number of points per (shortest) wavelength ${ }^{15}$, denoted by $N_{\mathrm{ppwl}}$. Unless indicated otherwise, $N_{\mathrm{ppwl}}=7$.

Due to circular geometries, the analytical values can be written in the form of multipole expansions, see [13, Eqn (A.1)] for the scattered field $p$ and [13, Eqn (A.4)] for the solid displacement $u$. These expressions are defined in terms of the coefficients $X_{n}=\left(a_{n}, b_{n}, c_{n}, d_{n}\right)^{t}$ at each level $n \in \mathbb{N}$, which satisfy the linear system $\mathbb{E}_{n} X_{n}=E_{n}$, see [13, Eqn (17)]. Since our problem OP differs from [12] in the $\mathrm{ABC}(19)$, our entries $\mathbb{E}_{n}^{41}$ and $\mathbb{E}_{n}^{42}$ are different, with

$$
\mathbb{E}_{n}^{4 i}=\kappa \mathrm{H}_{n}^{(i) \prime}(\kappa b)+\left(\frac{1}{2 b}-i \kappa\right) H_{n}^{(i)}(\kappa b), i=1,2 .
$$

Here, $\mathrm{H}_{n}^{(\bullet)}$ are the cylindrical Hankel functions. The components of right-hand side $E_{n} \in \mathbb{C}^{4}$ are given in [12, Eqn (19)-(21)]. The derivatives $\partial p, \partial u$ are calculated in the same manner, however with coefficients $\partial X_{n}=\left(\partial a_{n}, \partial b_{n}, \partial c_{n}, \partial d_{n}\right)$, given by

$$
\partial X_{n}=-\mathbb{E}_{n}^{-1}\left(\partial \mathbb{E}_{n}\right) X_{n}=-\mathbb{E}_{n}^{-1}\left(\partial \mathbb{E}_{n}\right) \mathbb{E}_{n}^{-1} E_{n}
$$

While the true analytical values are infinite sums ${ }^{16}$, those for numerical purposes are truncated versions with $N=2\lceil\kappa b\rceil+1$ modes (a standard choice $c f$. [13]). For illustrative purposes, we show the real part of $\partial_{\lambda} p$ at $\kappa a=7$ in Figure $3(\mathrm{a})$.
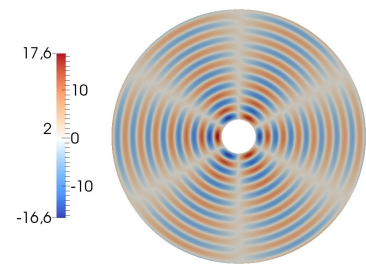

(a) Analytical value.

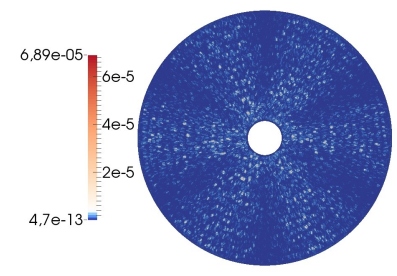

(b) Difference with IPDG.

Figure 3: Analytical value of $\operatorname{Re}\left(\partial_{\mu} p\right)$ at $\kappa a=7$ is shown in (a). Absolute difference with IPDG $\left|\operatorname{Re}\left(\partial_{\mu} p-\partial_{\mu} p_{h}\right)\right|=1.95 \times 10^{-7}$ is shown in (b), corresponding to $2.38 \times 10^{-5} \%$ relative error.

With the analytical values $p, u, \partial p, \partial u$ and the IPDG values $p_{h}, u_{h}, \partial p_{h}, \partial u_{h}$ calculated, we now obtain the analytical $p_{a, \infty}$ and numerical $\left(p_{h}\right)_{a, \infty}$ a-FFP and their corresponding derivatives. We recall that a-FPPs associated to problem $\mathbf{O P}$ are given by (37),

$$
\left[\begin{array}{c}
\left(p_{h}\right)_{a, \infty}(\hat{\mathbf{x}}) \\
p_{a, \infty}(\hat{\mathbf{x}})
\end{array}\right]=\mathfrak{c}(2) \int_{\Gamma}\left\{\Phi(\hat{\mathbf{x}}, \mathbf{y})\left[\begin{array}{c}
p_{h}(\mathbf{y}) \\
p(\mathbf{y})
\end{array}\right]-\left(\omega \rho_{\mathrm{f}}^{2}\left[\begin{array}{c}
u_{h}(\mathbf{y}) \\
u(\mathbf{y})
\end{array}\right] \cdot \nu-g(\mathbf{y})\right) \Psi(\hat{\mathbf{x}}, \mathbf{y})\right\} \mathrm{d} s(\mathbf{y})
$$

\footnotetext{
${ }^{15}$ Wavelength is given by $\frac{\text { propagation speed }}{\text { source frequency } f}$. Water has the shortest wavelength, due to its speed $\mathrm{c}_{\mathrm{f}}$ inferior to both the primary speed $\mathrm{c}_{\mathrm{P}}$ and the shear speed $\mathrm{c}_{\mathrm{S}}$ in steel as indicated in Table 1 .

16 They are uniformly convergent on compact subsets.
} 
with $g:=\frac{\partial p_{\mathrm{pw}}}{\partial \nu}$ and $\mathfrak{c}(2)$ defined in 13 , and their derivatives by 84 ,

$$
\left[\begin{array}{c}
\partial\left(p_{h}\right)_{a, \infty}(\hat{\mathbf{x}}) \\
\partial p_{a, \infty}(\hat{\mathbf{x}})
\end{array}\right]=\mathfrak{c}(2) \int_{\Gamma}\left\{\Phi(\hat{\mathbf{x}}, \mathbf{y})\left[\begin{array}{c}
\partial p_{h}(\mathbf{y}) \\
\partial p(\mathbf{y})
\end{array}\right]-\omega \rho_{\mathrm{f}}^{2}\left[\begin{array}{c}
\partial u_{h}(\mathbf{y}) \\
\partial u(\mathbf{y})
\end{array}\right] \cdot \nu \Psi(\hat{\mathbf{x}}, \mathbf{y})\right\} \mathrm{d} s(\mathbf{y}) .
$$

These quantities will be evaluated at 360 angles $\hat{\mathbf{x}}=\frac{\mathbf{x}}{|\mathbf{x}|}$, equally distributed on the unit circle. Here, square and curly braces group terms together, and do not have additional significance.

Remark 8 (a-FFP vs FFP). Recall that FFP can only be defined for the fluid pressure associated to problem $\mathbf{O P}_{\infty}$. If we consider a disc-shaped obstacle in this problem, the true FFP denoted by $p_{\infty}$ has the following expansion,

$$
p_{\infty}(\hat{\mathbf{x}})=\sum_{n} \sqrt{\frac{2}{\kappa \pi}} \frac{e^{i \pi / 4}}{\mathrm{i}^{n+1}} a_{n} \cos (n \theta), \hat{\mathbf{x}}=\frac{\mathbf{x}}{|\mathbf{x}|}
$$

where the coefficients $a_{n}$ are obtained by solving $\hat{\mathbb{E}}_{n} \hat{X}_{n}=\hat{E}_{n}$ with $\hat{X}_{n}=\left(a_{n}, c_{n}, d_{n}\right)^{t}$. These are obtained in the same way as $\mathbb{E}_{n}$ but without the $A B C 17$.

Experiments and observations. There are two main sets of comparison, both carried out for the normalized wavenumber range $4 \leq \kappa a \leq 12$. In the set 1 , Figure 4 and 5 show the comparison for $p, u$, and a-FPP, while in the second set, Figure 6 and 7 show that for their partial derivatives.

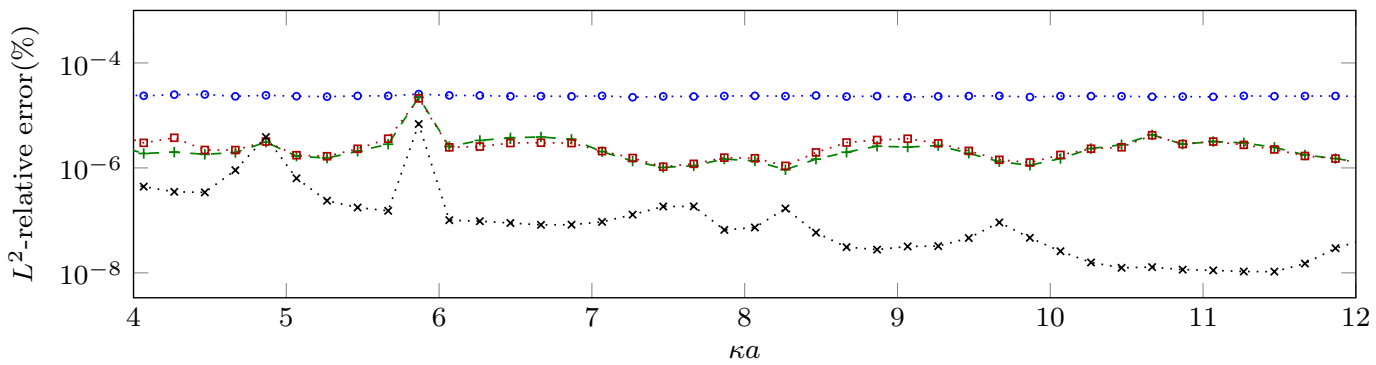

Figure 4: Comparison between the analytical and IPDG values for fluid pressure $\cdots \cdot \mathfrak{e}(p)$, solid displacement $-+-\mathfrak{e}\left(u_{x}\right), \cdots, \mathfrak{e}\left(u_{y}\right)$, and a-FFP $\cdots \in \mathfrak{e}\left(p_{a, \infty}\right)$.

Below are the observations for Figure 4

\footnotetext{
${ }^{17}$ In particular, $\hat{\mathbb{E}}_{n}$ is obtained from $\mathbb{E}_{n}$ removing the last row and second column, i.e.

$$
\hat{\mathbb{E}}_{n}=\left(\begin{array}{lll}
\mathbb{E}_{n}^{11} & \mathbb{E}_{n}^{13} & \mathbb{E}_{n}^{14} \\
\mathbb{E}_{n}^{21} & \mathbb{E}_{n}^{23} & \mathbb{E}_{n}^{24} \\
\mathbb{E}_{n}^{31} & \mathbb{E}_{n}^{33} & \mathbb{E}_{n}^{34}
\end{array}\right) \quad, \quad \hat{E}_{n}=\left(E_{n}^{1}, E_{n}^{2}, E_{n}^{3}\right)^{t}
$$
}




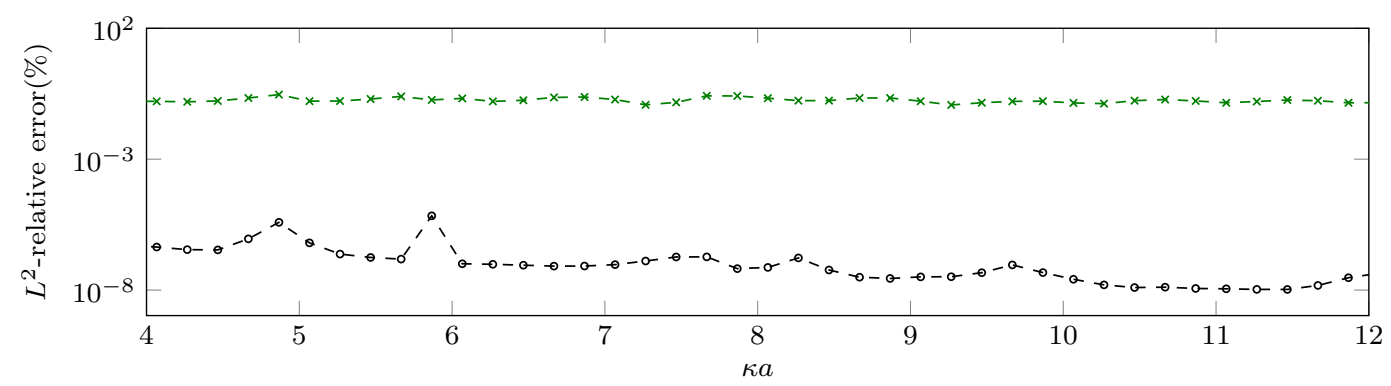

Figure 5: Error a-FFP-๑- $\mathfrak{e}\left(p_{a, \infty}\right)=\frac{\left|\left(p_{h}\right)_{a, \infty}-p_{a, \infty}\right|}{\left|p_{a, \infty}\right|} \times 100$ vs. error FFP $-*-\mathfrak{e}=\frac{\left|p_{\infty}-p_{a, \infty}\right|}{\left|p_{\infty}\right|} \times 100$. The error $\mathfrak{e}\left(p_{a, \infty}\right)$ is an indication of the quality of IPDG and the chosen integration quadrature, while $\mathfrak{e}$ reflects only the quality of the absorbing boundary condition in approximating the original problem $\mathbf{O P}_{\infty}$ on $\mathbb{R}^{2}$.

- Figure 4 shows that IPDG gives a very high accuracy level, with errors $\mathfrak{e}(p), \mathfrak{e}(u)$, $\mathfrak{e}\left(p_{a, \infty}\right)$ around $10^{-5 \%}$ for the current normalized wavenumber range. Although numerical a-FFP is calculated from one procedure of discretization (to calculate $u_{h}$ and $p_{h}$ ) and integration (97), we do not see the effect of accumulation of numerical error. Instead, the error $\mathfrak{e}\left(a_{p, \infty}\right)$ is lower than both $\mathfrak{e}(p)$ and $\mathfrak{e}(u)$.

- We see here the effectiveness of the mesh criteria defined in Remark 7, in maintaining the same error level, with the error curve either flat for $\mathfrak{e}(p)$ or varying in a fixed range for $\mathfrak{e}(u)$, instead of increasing with the frequency.

- With curved-boundary edge elements, the error curve $\mathfrak{e}(p)$ is flat and smooth, compared to those for solid displacement $\mathfrak{e}\left(u_{x}\right)$ and $\mathfrak{e}\left(u_{y}\right)$, which have spikes, especially around a Jones frequency ${ }^{18}$. This effect is also reported in [13].

- Since a-FFP is calculated in terms of both the traces of $p$ and $u$, its error curve $\mathfrak{e}\left(p_{a, \infty}\right)$ loses the flatness of $\mathfrak{e}(p)$, and takes on the fluctuation of $\mathfrak{e}(u)$.

The quality of the numerical solution also depends on the choice of the ABC. Figure 5 shows the satisfactory quality 19 of $\mathbf{T}_{1}$, by comparing the true FFP $p_{\infty}$, cf. (99), with the analytical a-FFP $p_{a, \infty}, c f$. (97), with $0.3 \%$ relative error.

We next comment on the second set of experiments given in Figures 6 - 7 , which show the errors for the partial derivatives, $\mathfrak{e}(\partial p), \mathfrak{e}(\partial u)$, and $\mathfrak{e}\left(\partial p_{a, \infty}\right)$.

\footnotetext{
${ }^{18}$ For an illustration of Jones frequencies, see [13, Fig.8], [38, Fig. 7].

${ }^{19}$ Although (satisfactorily) small, $\mathfrak{e}$ is much larger than the relative error $\mathfrak{e}\left(p_{a, \infty}\right)$. To decrease further the effect of the $\mathrm{ABC}$ (currently first order), higher orders can be used, and the radius $b$ of $\Omega_{\text {finite }}$ can be chosen larger.
} 
- Relative errors for both $\mathfrak{e}(\partial p)$ and $\mathfrak{e}(\partial u)$ are below than $10^{-4}, c f$. Figure 6(a)6(b). Although two discretization procedures are used (first to obtain $u_{h}$ and then the partial derivatives $), \mathfrak{e}(\partial p)$ and $\mathfrak{e}(\partial u)$ are around the same magnitude of $\mathfrak{e}(p)$ and $\mathfrak{e}(u)$ (shown $c f$. Figure 4). This means no effect of discretization error accumulation.

- The error $\mathfrak{e}\left(\partial p_{a, \infty}\right)$ in Figure 7 is slightly higher, fluctuating around $10^{-4} \%$. This slight increase is expected, since IPDG values of derivatives of a-FFP are computed from two discretization procedures (to obtain $\partial u_{h}$ and $\partial p_{h}$ ) and an integration procedure in 98 . However, accumulation of discretization error is still very negligible.

- Compared with the flat and smooth curve of $\mathfrak{e}(p)$, a small peak just before $\kappa a=6$ is now observed in that of $\mathfrak{e}(\partial p)$. Outside of this neighborhood, the error curve stays smooth and flat. For the solid displacement, this peak, already observed for the curve of $\mathfrak{e}(u)$, is now more pronounced for $\mathfrak{e}(\partial u)$.

- The error with respect to $\lambda$ behaves in the same way as with respect to $\mu$, apart from the following minute distinctions. Figure 7 shows that $\mathfrak{e}\left(\partial_{\mu} p_{a, \infty}\right)$ is always below $\mathfrak{e}\left(\partial_{\lambda} p_{a, \infty}\right)$, and in Figure 6(a) 6(b), the peak just before $\kappa a=6$ is more pronounced in $\mathfrak{e}\left(\partial_{\mu} p\right)$ than in $\mathfrak{e}\left(\partial_{\lambda} p\right)$.

\section{Conclusion}

For the stated fluid-solid interaction problem with a Lipschitz interface, we have shown the separate continuity and partial Fréchet differentiability of the solution operator and a-FFP with respect to the solid materials. The main difficulty in treating Lipschitz interfaces is the presence, in the PDE form of the auxiliary problem (which is used to characterize the partial derivatives), of inhomogeneous interface terms whose normal traces cannot be defined in canonical boundary Sobolev spaces. This is overcome in our work, with well-posedness shown in the standard framework for both the PDE and variational form (of the auxiliary problems). Joint continuity and full Fréchet differentiability results can be obtained with slight modification of the current work. Our analytical-numerical comparisons show that using IPDG equipped with curved boundary edge elements and high enough discretization order, in this characterization, gives high precision and incurs almost no effect of discretization error accumulation.

\section{Acknowledgement}

The authors would like to thank the referees for their constructive comments. The research of Ha Pham is supported by the E2S CHICKPEA project e2s_ uppa.eu/en/). 


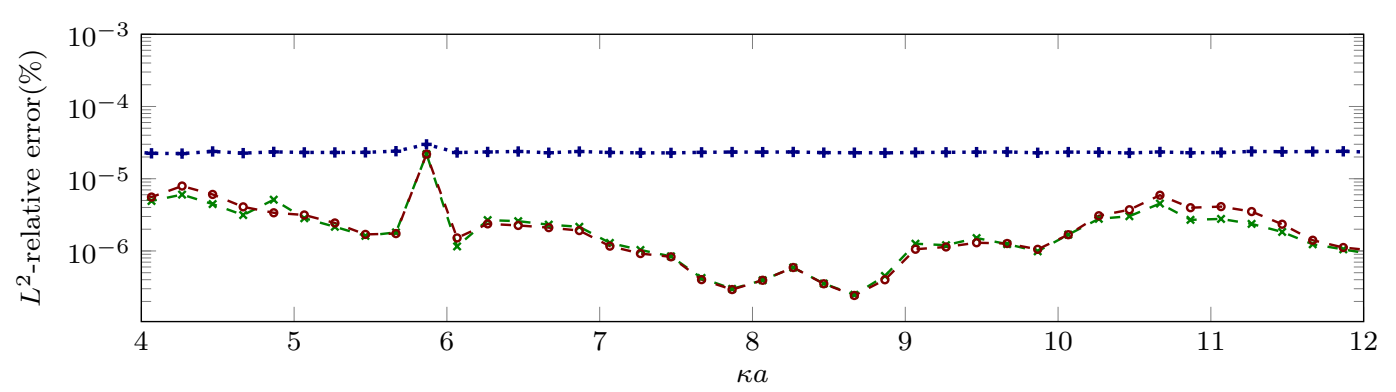

(a) Comparison for partial derivative with respect to $\lambda$.

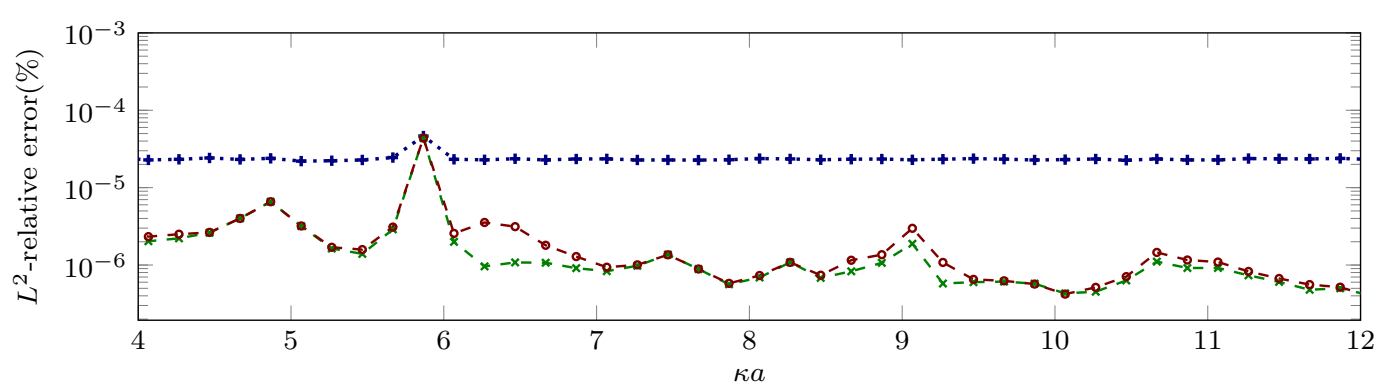

(b) Comparison for partial derivative with respect to $\mu$.

Figure 6: Comparison between the analytical and IPDG values of the Fréchet derivative of fluid pressure $\cdots+\cdots \mathfrak{e}(\partial p)$ and solid displacement $-*-\mathfrak{e}\left(\partial u_{x}\right),-\bullet \mathfrak{e}\left(\partial u_{y}\right)$.

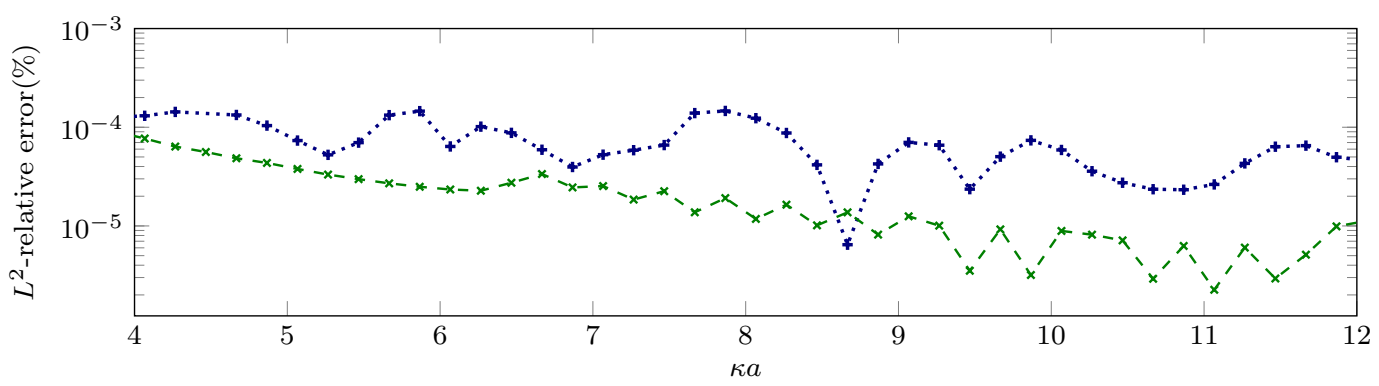

Figure 7: Comparison between the analytical and IPDG values of the Fréchet derivative of a-FFP. $\cdots+\cdots \mathfrak{e}\left(\partial_{\lambda} p_{a, \infty}\right),-*-\mathfrak{e}\left(\partial_{\mu} p_{a, \infty}\right)$.

\section{Bibliography}

[1] F. Hettlich, Fréchet derivatives in inverse obstacle scattering, Inverse problems 11 (2) (1995) 371.

[2] F. Le Louër, A domain derivative-based method for solving elastodynamic inverse obstacle scattering problems, Inverse Problems 31 (11) (2015) 115006.

[3] A. Kirsch, The domain derivative and two applications in inverse scattering theory, Inverse problems 9 (1) (1993) 81. 
[4] R. Potthast, Fréchet differentiability of the solution to the acoustic Neumann scattering problem with respect to the domain, Journal of Inverse and IllPosed Problems 4 (1) (1996) 67-84.

[5] R. Djellouli, C. Farhat, J. Mandel, P. Vanek, Continuous Fréchet differentiability with respect to a Lipschitz domain and a stability estimate for direct acoustic scattering problems, IMA journal of applied mathematics 63 (1) (1999) 51-69.

[6] F. L. Louër, On the Fréchet derivative in elastic obstacle scattering, SIAM Journal on Applied Mathematics 72 (5) (2012) 1493-1507.

[7] A. Charalambopoulos, On the Fréchet differentiability of boundary integral operators in the inverse elastic scattering problem, Inverse Problems 11 (6) (1995) 1137.

[8] S. Hubmer, E. Sherina, A. Neubauer, O. Scherzer, Lamé parameter estimation from static displacement field measurements in the framework of nonlinear inverse problems, arXiv preprint arXiv:1710.10446.

[9] E. Beretta, E. Francini, A. Morassi, E. Rosset, S. Vessella, Lipschitz continuous dependence of piecewise constant Lamé coefficients from boundary data: the case of non-flat interfaces, Inverse Problems 30 (12) (2014) 125005.

[10] A. Kirsch, A. Rieder, On the linearization of operators related to the full waveform inversion in seismology, Mathematical Methods in the Applied Sciences 37 (18) (2014) 2995-3007.

[11] A. Lechleiter, J. W. Schlasche, Identifying Lamé parameters from timedependent elastic wave measurements, Inverse Problems in Science and Engineering 25 (1) (2017) 2-26.

[12] H. Barucq, R. Djellouli, E. Estecahandy, On the existence and the uniqueness of the solution of a fluid-structure interaction scattering problem, Journal of Mathematical Analysis and applications 412 (2) (2014) 571-588.

[13] H. Barucq, R. Djellouli, E. Estecahandy, Efficient DG-like formulation equipped with curved boundary edges for solving elasto-acoustic scattering problems, International Journal for Numerical Methods in Engineering 98 (10) (2014) $747-780$.

[14] H. Barucq, R. Djellouli, E. Estecahandy, M. Moussaoui, Mathematical Determination of the Fréchet Derivative with Respect to the Domain for a FluidStructure Scattering Problem. Case of Polygonal-Shaped Domains, SIAM, Journal of Mathematical Analysis (2017) 1-29. 
[15] H. Barucq, R. Djellouli, E. Estecahandy, Characterization of the Fréchet derivative of the elasto-acoustic field with respect to Lipschitz domains, Journal of Inverse and Ill-Posed Problems 22 (1) (2014) 1-8.

[16] H. Barucq, R. Djellouli, E. Estecahandy, Fréchet differentiability of the elastoacoustic scattered field with respect to Lipschitz domains, Mathematical Methods in the Applied Sciences 40 (2) (2017) 404-414.

[17] R. Potthast, Fréchet differentiability of boundary integral operators in inverse acoustic scattering, Inverse Problems 10 (2) (1994) 431.

[18] M. Costabel, F. Le Louër, Shape derivatives of boundary integral operators in electromagnetic scattering. part I: Shape differentiability of pseudohomogeneous boundary integral operators, Integral Equations and Operator Theory 72 (4) (2012) 509-535.

[19] F. Le Louër, Optimisation de forme d'antennes lentilles intégrées aux ondes millimétriques, Ph.D. thesis, Université Européenne de Bretagne (2009).

[20] R. Potthast, Domain derivatives in electromagnetic scattering, Mathematical Methods in the Applied sciences 19 (15) (1996) 1157-1175.

[21] L. Päivärinta, R. Kress, On the far field in obstacle scattering, SIAM Journal on Applied Mathematics 59 (4) (1999) 1413-1426.

[22] H. Haddar, R. Kress, On the Fréchet derivative for obstacle scattering with an impedance boundary condition, SIAM Journal on Applied Mathematics 65 (1) (2004) 194-208.

[23] T. Hohage, Iterative methods in inverse obstacle scattering: regularization theory of linear and nonlinear exponentially ill-posed problems, Ph.D. thesis, Johannes-Kepler-Universitat Linz (1999).

[24] C. J. Alves, R. Kress, On the far-field operator in elastic obstacle scattering, IMA Journal of Applied Mathematics 67 (1) (2002) 1-21.

[25] W. C. H. McLean, Strongly elliptic systems and boundary integral equations, Cambridge university press, 2000.

[26] S. A. Sauter, C. Schwab, Boundary element methods, Springer, 2010.

[27] P. Monk, V. Selgas, An inverse fluid-solid interaction problem, Inverse Problems \& Imaging 3 (2) (2009) 173-198. 
[28] G. C. Hsiao, R. E. Kleinman, G. F. Roach, Weak solutions of fluid-solid interaction problems, Mathematische Nachrichten 218 (1) (2000) 139-163.

[29] A. Buffa, Trace theorems on non-smooth boundaries for functional spaces related to Maxwell equations: an overview, Lecture notes in computational science and engineering 28 (2003) 23-34.

[30] D. Colton, R. Kress, Inverse acoustic and electromagnetic scattering theory, 3rd Edition, Vol. 93, Springer Science \& Business Media, 2012.

[31] F. Ihlenburg, Finite element analysis of acoustic scattering, Vol. 132, Springer Science \& Business Media, 2006.

[32] X. Antoine, H. Barucq, A. Bendali, Bayliss-Turkel-like radiation conditions on surfaces of arbitrary shape, Journal of Mathematical Analysis and Applications 229 (1) (1999) 184-211.

[33] X. Antoine, Conditions de radiation sur le bord, Ph.D. thesis, Université de Pau et Pays de l'Adour. (1997).

[34] F. Cakoni, D. Colton, Qualitative methods in inverse scattering theory: An introduction, Springer Science \& Business Media, 2005.

[35] D. Baskin, E. A. Spence, J. Wunsch, Sharp high-frequency estimates for the Helmholtz equation and applications to boundary integral equations, SIAM Journal on Mathematical Analysis 48 (1) (2016) 229-267.

[36] A. Kimeswenger, O. Steinbach, G. Unger, Coupled finite and boundary element methods for fluid-solid interaction eigenvalue problems, SIAM Journal on Numerical Analysis 52 (5) (2014) 2400-2414.

[37] P. Monk, E. Süli, The adaptive computation of far-field patterns by a posteriori error estimation of linear functionals, SIAM Journal on Numerical Analysis 36 (1) (1998) 251-274.

[38] T. Huttunen, J. Kaipio, P. Monk, An ultra-weak method for acoustic fluidsolid interaction, Journal of Computational and Applied Mathematics 213 (1) (2008) 166-185. 